Spring 5-9-2020

\title{
An Evaluation of a Renewal-Mitigation Procedure for Inappropriate Mealtime Behavior
}

\author{
Sarah D. Haney \\ University of Nebraska Medical Center
}

Tell us how you used this information in this short survey.

Follow this and additional works at: https://digitalcommons.unmc.edu/etd

Part of the Applied Behavior Analysis Commons

\section{Recommended Citation}

Haney, Sarah D., "An Evaluation of a Renewal-Mitigation Procedure for Inappropriate Mealtime Behavior" (2020). Theses \& Dissertations. 457.

https://digitalcommons.unmc.edu/etd/457

This Dissertation is brought to you for free and open access by the Graduate Studies at DigitalCommons@UNMC. It has been accepted for inclusion in Theses \& Dissertations by an authorized administrator of DigitalCommons@UNMC.For more information, please contact digitalcommons@unmc.edu. 


\title{
AN EVALUATION OF A RENEWAL-MITIGATION PROCEDURE FOR INAPPROPRIATE MEALTIME BEHAVIOR
}

\author{
by
}

\section{Sarah Diane Haney}

\author{
A DISSERTATION \\ Presented to the Faculty of the University of \\ Nebraska Graduate College \\ in Partial Fulfillment of the Requirements \\ For the Degree of Doctor of Philosophy
}

Medical Sciences Interdepartmental Area Graduate Program

(Applied Behavior Analysis)

Under the Supervision of Professor Cathleen C. Piazza

University of Nebraska Medical Center

Omaha, Nebraska

April, 2020

Supervisory Committee:

Cathleen C. Piazza, Ph.D.

Kathryn M. Peterson, Ph.D.

Bethany A. Hansen, Ph.D.

Brian D. Greer, Ph.D 


\section{ACKNOWLEDGMENTS}

My family, especially my parents and siblings, who have loved, supported, and encouraged me my entire life. My parents instilled in me a strong work ethic, passion for learning, and encouraged me to always believe in myself and pursue my dreams. I am grateful for their unconditional love and support.

Dr. Piazza, who has been an incredible mentor to me during this program. Her passion for and dedication to children and families we serve is inspiring. Her contributions to our field have forever changed lives around the world, including mine. Dr. Peterson, who has been a second mentor to me during this program. Her intelligence, guidance, leadership, support, and clinical expertise have been essential to my success in this program. Thank you both for shaping me into a scientist-practitioner and for cultivating my passion for serving children with feeding disorders.

My feeding family at the Munroe-Meyer Institute's Pediatric Feeding Disorders Program, who assisted me with this study and supported me during my research and clinical endeavors. My feeding gals and fellow doctorate students who became my best friends and colleagues. Kayla Randall, for keeping me motivated throughout this program. Thank you all for your advice, support, humor, friendship, and for celebrating my accomplishments with me. Thank you to the families who allowed me to work with their children and celebrate their feeding milestones with my praise parties.

Lastly, I would like to thank my best friend, the love of my life, and future husband, JT. Thank you for believing in me, encouraging me, moving across the country with me, feeding me, dancing and singing with me, and making me laugh. I cannot express how grateful I am for the love and support you showed me every second of this program. You deserve an honorary doctorate for your patience, positivity, and encouragement during our journey in this program. Thank you for always encouraging me to follow my dreams. I look forward to our next adventures and to celebrating our future accomplishments together. 


\title{
AN EVALUATION OF A RENEWAL-MITIGATION PROCEDURE FOR INAPPROPRIATE MEALTIME BEHAVIOR
}

\author{
Sarah D. Haney, Ph.D. \\ University of Nebraska, 2020
}

Supervisor: Cathleen C. Piazza, Ph.D.

Renewal, the increase in problem behavior during extinction following a context change (e.g., transfer of intervention from clinic to home), may be particularly concerning during interventions for feeding disorders because multiple context changes are often necessary for intervention generality and maintenance (Podlesnik et al., 2017). In the current study, we tested for renewal and evaluated a renewal-mitigation procedure when we transferred the intervention from a therapist to a caregiver, from clinic to the home, and introduced Context A foods. We used an ABA arrangement to evaluate the generality of the renewal effect with 7 children with feeding disorders. Context A was functional reinforcement. Context B was function-based extinction during the control and mitigation conditions and our renewal-mitigation procedure in the mitigation condition. The renewal test was function-based extinction in Context A. We observed renewal of inappropriate mealtime behavior following an implementer, setting, and implementer plus setting change. Of the children for whom we observed renewal, our renewal-mitigation procedures were effective for 4 of 4 children. 


\section{TABLE OF CONTENTS}

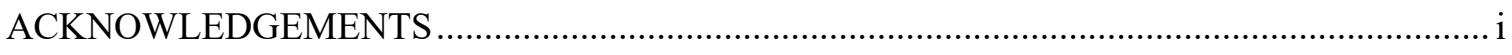

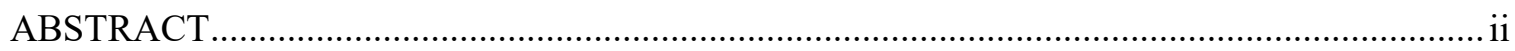

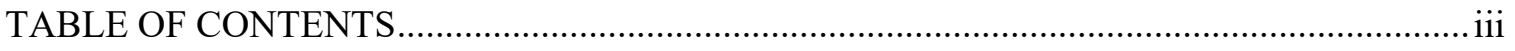

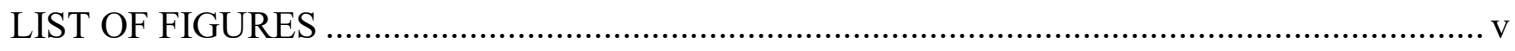

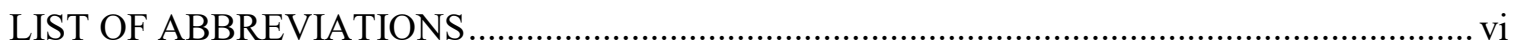

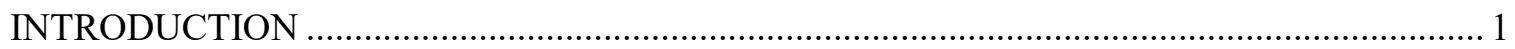

Treatmemt of Pediatric Feeding Disorders..................................................................... 1

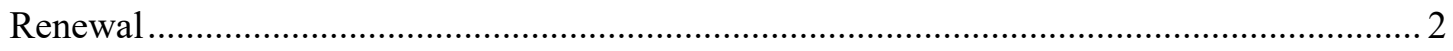

Clinical Implications of Renewal........................................................................... 3

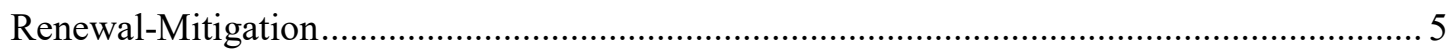

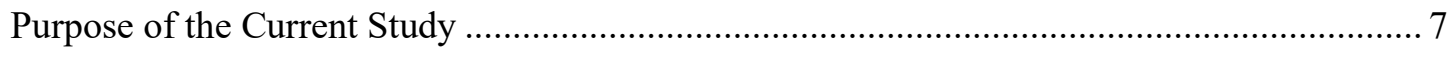

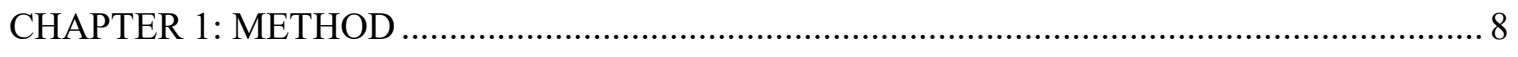

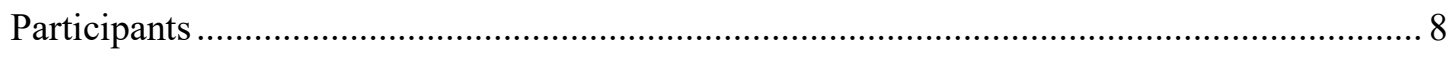

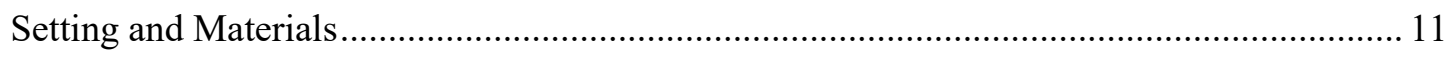

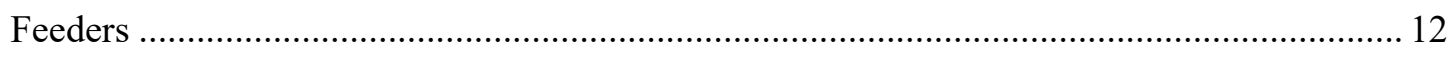

Dependent Variables, Procedural Integrity, and Interobserver Agreement............................ 12

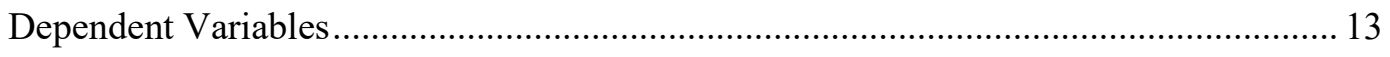

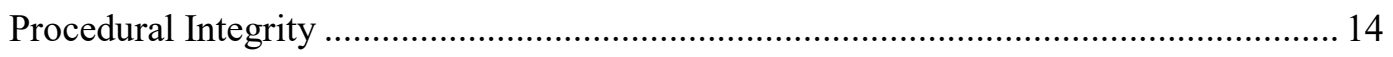

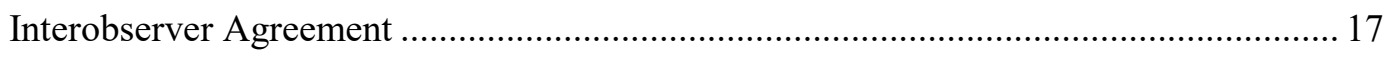

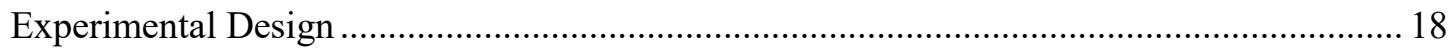

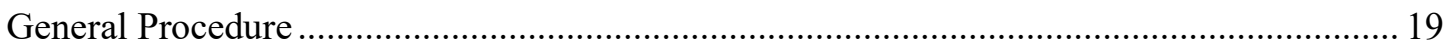

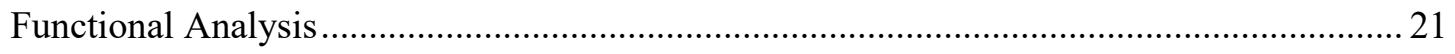

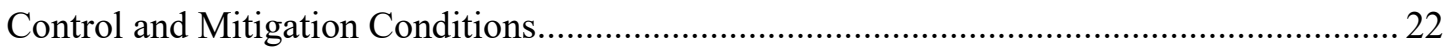

Context A Reinforcement (Control and Mitigation Conditions) ….................................. 24

Context B Extinction (Control Condition) ………......................................................... 24 
Caregiver Training (Control Condition) ..................................................... 25

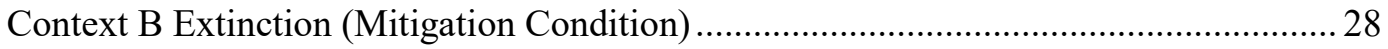

Caregiver Training and Mitigation Procedure (Mitigation Condition)................ 28

Context A Extinction (Renewal Test, Control and Mitigation Conditions)...................... 31

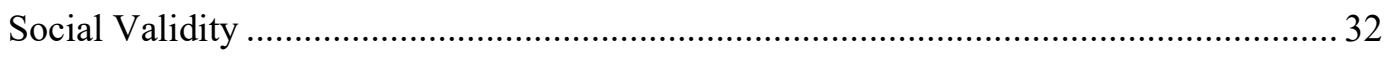

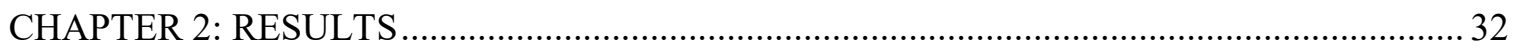

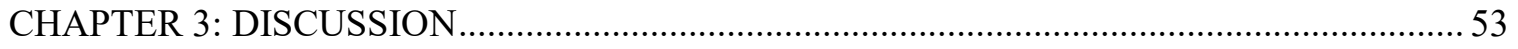

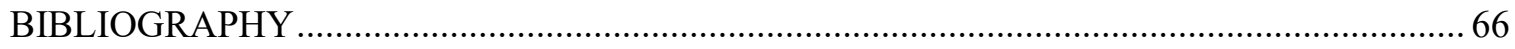




\section{LIST OF FIGURES}

Figure 1. Rate of Inappropriate Mealtime Behavior for John, Maisy, Hope, and Emilia ................ 1

Figure 2. Rate of Inappropriate Mealtime Behavior for Diego................................................ 1

Figure 3. Rate of Inappropriate Mealtime Behavior for Jade. .................................................. 1

Figure 4. Rate of Inappropriate Mealtime Behavior for Julian................................................. 1

Figure 5. Percentage of Acceptance for John, Maisy, Hope, and Emilia....................................... 1

Figure 6. Percentage of Acceptance for Diego................................................................... 1

Figure 7. Percentage of Acceptance for Jade ....................................................................... 1

Figure 8. Percentage of Acceptance for Julian. ..................................................................... 1

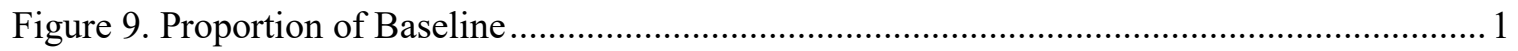




\section{LIST OF ABBREVIATIONS}

ABA (Renewal) context A reinforcement, context B extinction, context A extinction $\mathrm{ABC}$ (Renewal) context $\mathrm{A}$ reinforcement, context $\mathrm{B}$ extinction, context $\mathrm{C}$ extinction $\mathrm{AAB}$ (Renewal) context A reinforcement, context A extinction, context B extinction ABA applied behavior analysis 


\section{INTRODUCTION}

\section{Treatment of Pediatric Feeding Disorders}

Approximately one in four children with normative development and eight in 10 children with a developmental disability exhibit feeding disorders or avoidant/restrictive food intake disorder (University of Rochester Medical Center, 2019). Professionals use the diagnosis avoidant/restrictive food intake disorder to describe children who do not consume enough calories or nutrients to sustain weight or grow (DSM-5; American Psychiatric Association, 2013). Insufficient calories or nutrients may have negative short-and long-term consequences like poor growth and behavior and learning problems, respectively (Freedman et al., 1999; Peterson et al., 2018; Volkert \& Piazza, 2012). Pediatric feeding disorders like avoidant/restrictive food intake disorder also may cause anxiety, depression, stress, and strained caregiver-child relationships (Bryant-Waugh et al., 2010; Kreipe \& Palomaki, 2012; Volkert et al., 2016; Volkert \& Piazza, 2012).

Although the etiology of avoidant/restrictive food intake disorder is complex and multifaceted (Volkert \& Piazza, 2012), researchers hypothesize that environmental events, like gagging, choking, or vomiting during feeding, may be responsible for the development and maintenance of inappropriate mealtime behavior at least partially (Borerro et al., 2010; Piazza, Fisher, et al., 2003). The child may engage in inappropriate mealtime behavior, like batting at the spoon or head turning, to avoid bite or drink presentations. Research has shown and we observe clinically that caregivers of children with avoidant/restrictive food intake disorder and feeding disorders, respectively, provide escape from bite or drink presentations; meal termination; attention; and access to tangible items, like toys or preferred food or drink when the child engages in inappropriate mealtime behavior (Borrero et al., 2010; Piazza, Fisher, et al., 2003). In addition, results of several studies have shown that these caregiver-provided consequences may function as reinforcement for inappropriate mealtime behavior (Bachmeyer, et al., 2009; Bachmeyer et al., 
2019; Girolami \& Scotti, 2001; Najdowski et al., 2008; Piazza, Fisher, et al., 2003). For example, Piazza et al. (2017) analyzed data from 38 functional analyses of inappropriate mealtime behavior and found that escape from bites or drinks functioned as at least one of the reinforcers for inappropriate mealtime behavior for $85 \%$ of children.

Given the prevalence that escape functions as reinforcement for inappropriate mealtime behavior, the effectiveness of escape extinction as treatment is not surprising. Escape-extinction interventions like nonremoval of the spoon or physical guidance are the most established and empirically supported intervention for feeding disorders (Volkert \& Piazza, 2012). Although researchers have demonstrated the effectiveness of escape extinction in clinical settings, less is known about the generalization and long-term maintenance of escape extinction outside of the clinical setting.

\section{Renewal}

Researchers have identified context as one variable that may affect the generality and long-term maintenance of the effects of extinction (e.g., Kelley et al., 2015; Podlesnik et al., 2017). Context refers to any stimulus that exerts control over behavior and includes exteroceptive stimuli such as odor, light, color, tactile, and the experimental chamber (e.g., Bouton et al., 2011; Kelley et al., 2015; Podlesnik et al., 2017; Todd, 2013) and environmental stimuli such as the individual who implements extinction, the location in which an individual experiences extinction (e.g., home, school), and the passage of time (e.g., Ibañez et al., 2019; Kelley et al., 2018; Podlesnik et al., 2017; Saini et al., 2018). Researchers hypothesize that context changes may be responsible for increases in previously extinguished behavior, a relapse phenomenon called renewal (Bouton et al., 2011; Kelley et al., 2015; Podlesnik et al., 2017). Context theory states that renewal occurs because the context functions as a discriminative stimulus for the extinction contingencies, and the effects of these discriminative stimuli are specific to the original extinction context. Thus, the effects of extinction do not have generality outside of the original extinction 
context (Bouton et al., 2011; Podlesnik et al., 2017; Trask et al., 2017; Wathen \& Podlesnik, 2018), perhaps because the specific stimuli that were present in the original extinction context are not present when the extinction context changes (Wathen \& Podlesnik, 2018).

Researchers have demonstrated renewal in basic and applied preparations using ABA, $\mathrm{ABC}$, and $\mathrm{AAB}$ arrangements (e.g., Bouton et al., 2011; Cohenour et al., 2018; Ibañez et al., 2019; Kelley et al., 2015; Kelley et al., 2018; Liddon et al., 2018; Mitteer et al., 2018; Saini et al., 2018; Todd, 2013; Todd et al., 2012). The ABA arrangement, however, has produced the most robust renewal effects (Bouton et al., 2011). In ABA renewal, the researcher provides reinforcement for a target behavior, like inappropriate mealtime behavior, in Context A, such as clinic Room 1, and implements extinction for inappropriate mealtime behavior in Context B, such as clinic Room 2. The renewal test is a return to Context A in clinic Room 1 where the researcher continues to implement extinction for inappropriate mealtime behavior. The return to Context A in clinic Room 1 represents a change from the context in which the researcher implemented extinction initially, Context B in clinic Room 2, to the context in which the participant has a history of reinforcement for inappropriate mealtime behavior, Context A in clinic Room 1. Renewal occurs if the level of inappropriate mealtime behavior increases during the renewal test in Context A in clinic Room 1 above the level of inappropriate mealtime behavior in Context B in clinic Room 2.

\section{Clinical Implications of Renewal}

The study of renewal is particularly relevant during treatment of children with avoidant/restrictive food intake disorder for several reasons. First, context changes are common because children feed in many environments with many different individuals. Second, inappropriate mealtime behavior that emerges during context changes may be more likely to contact reinforcement, which may maintain the behavior over time (Ibañez et al., 2019; Kelley et al., 2018; Mitteer et al., 2018). Third, studying renewal may inform strategies to improve the 
generality and long-term maintenance of intervention effects (Ibañez et al., 2019; Kelley et al., 2018; Podlesnik et al., 2017; Wathen \& Podlesnik, 2018).

Our standard clinical practice treating children with avoidant/restrictive food intake disorder provides us with an opportunity to study renewal in this population. Caregivers report and we observe during our initial evaluation of the child that the caregiver provides reinforcement for inappropriate mealtime behavior. When we admit the child, we observe the caregiver feeding the child in the clinic using the consequences he or she provides at home, which we define as Context A. Because children admitted to our program have complex feeding problems, trained therapists often implement the child's intervention initially. Thus, Context B is the therapist implementing extinction in the clinic. After the therapist demonstrates the intervention's efficacy, we train caregivers to implement the intervention in the clinic. The return to Context $\mathrm{A}$ is the caregivers implementing extinction in the clinic. After caregivers are implementing the intervention in the clinic with greater than $80 \%$ integrity, we observe the caregivers implementing the intervention in the home. Thus, we have an additional context change, which is the transition from the caregiver feeding in the clinic to the caregiver feeding at home. More context changes may be necessary if the child feeds in other environments, like school or daycare, or with other caregivers, like teachers or babysitters, or we increase the child's diet variety or change the texture of presented food.

Two studies have demonstrated renewal during feeding interventions (Ibañez et al., 2019; Kelley et al., 2018). Both studies used an ABA arrangement to evaluate renewal of inappropriate mealtime behavior during function-based extinction after changes in the feeder (Kelley et al., 2018) and feeder and setting (Ibañez et al., 2019) for children diagnosed with a feeding disorder. Ibañez et al. (2019) conducted functional analyses of the inappropriate mealtime behavior of four children. Context A was functional reinforcement of inappropriate mealtime behavior in a simulated home setting with the child's caregiver as feeder. Context B was function-based 
extinction in a standard clinic room with a therapist as feeder. The return to Context A was function-based extinction in a simulated home setting with the caregiver as feeder. Returning to Context A produced renewal of inappropriate mealtime behavior for the three children even though caregivers implemented function-based extinction with high levels of integrity.

We could find no additional studies that examined renewal of inappropriate mealtime behavior with children with avoidant/restrictive food intake disorder. Thus, we do not know how often it occurs. To gain a better estimate of how often renewal of inappropriate mealtime behavior occurs, we analyzed data from 24 children with feeding disorders admitted sequentially to our pediatric feeding disorders day-treatment program from 2017 to 2018. We included the data in our analysis if we had exposed the child to a function-based extinction and a context change, which produced 57 cases. We used the criterion Kelley et al. (2018) described to determine which cases demonstrated renewal. We found that renewal of inappropriate mealtime behavior occurred during $44 \%$ of cases in which the feeder changed from therapist to caregiver and $53 \%$ of cases in which the setting changed from clinic to home. Similarly, Muething et al. (2020) analyzed data from children admitted to a day treatment program for severe problem behavior from 2010-2015 and found that renewal of severe problem behavior occurred during $42 \%$ of cases following an implementer change (e.g., therapist to therapist) and $42 \%$ of cases following a setting change (e.g., clinic to home). Given the high prevalence of renewal during extinction for socially significant behavior, it is important for clinicians to evaluate preventative procedures for renewal in clinical settings.

\section{Renewal-Mitigation}

Our analysis of cases that we exposed to function-based extinction and a context change suggests renewal of inappropriate mealtime behavior may occur after changes to the intervention context in over $50 \%$ of cases. We also know that context changes are likely for children with avoidant/restrictive food intake disorder. Thus, developing and testing procedures to mitigate 
renewal is important. Researchers have tested several procedures to mitigate the renewal of operant behavior such as implementing the intervention initially in multiple contexts (BandarianBalooch \& Neumann, 2011; Bernal-Gamboa, Nieto, et al., 2017; Kelley et al., 2018; Krisch et al., 2018), increasing the similarity of the intervention contexts (Bandarian-Balooch \& Neumann, 2011; Todd et al., 2012), and introducing discriminative stimuli in the extinction-intervention context and using these discriminative stimuli in other intervention contexts (Bernal-Gamboa, Gámez, et al., 2017; Nieto et al., 2017; Todd et al., 2012).

Although researchers have assessed the renewal-mitigation procedures described above with operant behavior, we could find only one study that assessed renewal-mitigation with children with socially significant behavior. Kelley et al. (2018) used an ABABA+BA arrangement to evaluate renewal and a renewal-mitigation procedure for inappropriate mealtime behavior following a change in the feeder from therapist to caregiver during extinction intervention for two children with avoidant/restrictive food intake disorder. Inappropriate mealtime behavior returned for both children during the first renewal test when caregivers implemented extinction. The researchers returned to Context $\mathrm{B}$, in which the therapist implemented function-based extinction. Context $\mathrm{A}+\mathrm{B}$ was their renewal-mitigation procedure in which the caregiver sat next to the therapist while the therapist implemented function-based extinction. During the second renewal test in Context A when the caregiver implemented function-based extinction alone, inappropriate mealtime behavior remained at zero for one participant and occurred for two sessions before decreasing to zero for the other participant; however, rate of inappropriate mealtime behavior was lower and less persistent following the mitigation procedure. Results demonstrated that pairing the caregiver with cues associated with extinction (e.g., therapist) was sufficient to mitigate renewal of inappropriate mealtime behavior. One limitation of the study was that the researchers evaluated renewal and their renewalmitigation procedure sequentially using a reversal design to test for renewal and evaluate their 
renewal-mitigation procedure. Results of basic research have shown that repeated exposure to a context change, like in the reversal design Kelley et al. used, may reduce the likelihood that o behavior will return (Sweeney \& Shahan, 2013; Wacker et al., 2011). Thus, we cannot be certain that the absence of renewal in the return to Context A after the renewal-mitigation procedure was due to the renewal-mitigation procedure or due to repeated exposure to the context change. A second limitation is that although Kelley et al. used a bug-in-the-ear device to provide feedback to caregivers while they were implementing the intervention, they did not collect data on intervention integrity.

\section{Purpose of the Current Study}

Additional studies are needed to evaluate renewal and renewal-mitigation procedures for children with avoidant/restrictive food intake disorder given the limited research on this topic and the high likelihood that renewal occurs with these children. In the current study, we sought to extend the findings of Ibañez et al. (2019) by evaluating renewal of inappropriate mealtime behavior during function-based extinction in the control condition. We also sought to extend the findings of Kelley et al. (2018) by developing and evaluating a renewal-mitigation procedure in our mitigation condition. Results of previous research have shown that renewal may be less likely when cues for extinction of the target behavior, like the therapist feeding the child, are present in contexts previously associated with reinforcement of the target behavior, like the caregiver feeding the child (Bernal-Gamboa, Gámez, et al., 2017; Kelley et al., 2018; Nieto et al., 2017; Todd et al., 2012). Researchers have also shown that increasing the similarity between acquisition (i.e., reinforcement) and extinction contexts may mitigate renewal when returning to contexts associated with reinforcement (Bandarian-Balooch \& Neumann, 2011; Todd et al., 2012). In addition, combining renewal-mitigation procedures may mitigate renewal more effectively than these strategies individually (Bandarian-Balooch \& Neumann, 2011; Bernal-Gamboa, Nieto, et al., 2017; Krisch et al., 2018; Podlesnik et al., 2017). Thus, our renewal-mitigation procedure 
used a therapist-caregiver pairing procedure like Kelley et al. to prevent renewal following a change in feeder from therapist to caregiver. We used the therapist-caregiver pairing procedure and a food fading procedure to prevent renewal following a change in feeder and food with one child. We used the therapist-caregiver pairing procedure and increased the similarity of the session room to the home to prevent renewal following a change in feeder and setting with one child and increased the similarity of the session room to a simulated home to prevent renewal following a setting change for one child.

We assessed renewal and renewal-mitigation during changes in the feeder, food, and setting, using an ABA renewal arrangement. Context $\mathrm{A}$ was the caregiver or therapist feeding the child and delivering function-based reinforcement for inappropriate mealtime behavior in the control and mitigation conditions. Context $\mathrm{B}$ was the therapist feeding the child and implementing function-based extinction in the control and mitigation conditions and implementing the renewal-mitigation procedure in the mitigation condition. We evaluated rate of inappropriate mealtime behavior in a return to Context $\mathrm{A}$ in which the caregiver or therapist fed the child using function-based extinction in the control and mitigation conditions.

\section{CHAPTER 1: METHOD}

\section{Participants}

Participants were children with feeding disorders admitted to an intensive day-treatment program and their caregivers. Children attended the program 5 days per week from approximately 9:00 a.m. to 5:00 p.m. Inclusion criteria were that the child (a) was between 2 and 18 years old; (b) was safe for oral feeding per the child's pediatrician or a speech and language pathologist; (c) received $50 \%$ or more of his or her daily caloric needs from supplemental feeding like a gastrostomy tube or a liquid diet or had a diet that was significantly deficient relative to macronutrients, micronutrients, or both per our program's registered dietitian; (d) had received at 
least 3 months of outpatient feeding therapy and had not progressed or progress had plateaued; and (e) did not pack food or liquid in the mouth. Our program's registered dietician used the Centers for Disease Control and Prevention (2010) growth chart for ages 2-20 to calculate bodymass index, height, and weight for all children except Jade.

John was a 6-year-old boy whose diagnoses included autism spectrum disorder and avoidant/restrictive food intake disorder. He consumed yogurt, small amounts of fruit baby food, pudding, Pediasure 1.0, and no proteins, starches, or vegetables. At the time of admission, John received $114 \%, 348 \%$, and $136 \%$ of his daily caloric, protein, and fluid needs, respectively. He was in the $33^{\text {rd }}, 12^{\text {th }}$, and $17^{\text {th }}$ percentile for height, weight, and body-mass index, respectively, Our program's registered dietician estimated that John's caloric and macronutrient intake was adequate primarily due to his consumption of yogurt and Pediasure 1.0, but his diet was deficient in chromium, iron, manganese, potassium, selenium, and vitamins E and B3.

Maisy was a 4-year-old girl with a diagnosis of Ehlers-Danlos syndrome and a history of food allergies, gastrostomy-tube dependence, failure to thrive, poor weight gain, and resolved Food Protein Induced Enterocolitis Syndrome and milk, soy, protein, intolerance. She consumed water, 3 to $5 \mathrm{oz}$ of Puramino via a bottle, and small amounts of beans, eggs, peaches, chips, bread, macaroni $n$ cheese, and hotdog. At the time of admission, Maisy received $43 \%, 204 \%$, and $32 \%$ of her daily caloric, protein, and fluid needs, respectively via 14 to $16-\mathrm{oz}$ tube feedings of Vanilla Puramino Junior. She was in the $<1^{\text {st }},<1^{\text {st }}$, and $1^{\text {st }}$ percentile for height, weight, and bodymass index, respectively. Our program's registered dietician estimated that Emilia's intake was deficient in calcium, chromium, copper, magnesium, manganese, potassium, and vitamins B5 and D.

Hope was a 5-year-old girl with a history of developmental delay; resolved neurological defect with facial drooping; and nasojejunal-, gastrostomy-, and gastrostomy-jejunostomy-tube dependence. She consumed small amounts of chicken nuggets, steak, French fries, bread, 
potatoes, pineapple, apple, orange, applesauce, chocolate ice cream, juice, and chocolate milk. Hope received $84 \%, 160 \%$, and $40 \%$ of her daily caloric, protein, and fluid needs, respectively via 150-ml and 750-ml gastrostomy-jejunostomy-tube feedings of Peptamin Junior 1.5 once per day for a total of $900 \mathrm{ml}$. She was in the $7^{\text {th }}, 3^{\text {rd }}$, and $13^{\text {th }}$ percentile for height, weight, and bodymass index, respectively. Our program's registered dietician estimated that Hope's oral intake was deficient in Biotin; Folate; and Vitamins A, B1 Thiamine, B2 Riboflavin, B6, B12, C, D, and E.

Emilia was a 5-year-old girl with a history of gagging and vomiting, oral-motor-skill deficits with difficulty swallowing, and a soy allergy and a diagnosis of avoidant/restrictive food intake disorder. She consumed Ensure, Pediasure 1.0, juice, and small amounts of junk food like chips, cookies, crackers, donuts, rice cakes, but no fruits, proteins, starches, or vegetables. Emilia received $84 \%, 160 \%$, and $40 \%$ of her daily caloric, protein, and fluid needs, respectively. She was in the $68^{\text {th }}, 32^{\text {nd }}$, and $12^{\text {th }}$ percentile for height, weight, and body-mass index, respectively. Our program's registered dietician estimated that Emilia's intake was deficient in calcium, iron, manganese, potassium, and vitamin D.

Diego was a 3-year-old boy with a history and current status of total parenteral nutrition dependence and diagnoses of avoidant/restrictive food intake disorder, intestinal failure, and short-bowel syndrome. He consumed $59 \mathrm{ml}$ of Neocate Jr. from a bottle approximately every 2 hrs while awake and small amounts of goldfish crackers and peanut butter balls, but no fruit, proteins, starches, or vegetables. At the time of admission, Diego received $80 \%, 221 \%$, and $91 \%$ of his daily caloric, protein, and fluid needs, respectively. He was in the $19^{\text {th }}, 40^{\text {th }}$, and $68^{\text {th }}$ percentile for height, weight, and body-mass index, respectively. Our program's registered dietician estimated that his micronutrient intake was adequate due to his total parenteral nutrition and consumption of Neocate Jr. 
Jade was a 4-year-old girl with a history of a congenital heart defect; gastrostomy-tube dependence, dysphagia, gastroesophageal reflux disease, Nissen fundoplication, and oral-motorskill deficits and diagnoses of avoidant/restrictive food intake disorder, DiGeorge syndrome (22q11 deletion syndrome), and gastrostomy tube. She did not consume any fruits, proteins, starches, or vegetables orally. At the time of admission, Jade received $73 \%, 210 \%$, and $60 \%$ of her daily caloric, protein, and fluid needs, respectively, via $118 \mathrm{ml}$ gastrostomy-tube feedings of caregiver-blended foods 6 times per day for a total of $710 \mathrm{ml}$. She was in the $14^{\text {th }} 7^{\text {th }}$, and $9^{\text {th }}$ percentile for height, weight, and body-mass index, respectively, based on the 22q11 deletion syndrome growth chart for girls ages 2-20 (Tarquinio et al., 2012). Our program's registered dietician estimated that her intake was deficient in calcium, selenium, and vitamin D.

Julian was a 5-year-old male with a diagnosis of autism spectrum disorder. He consumed juice, water, cereal, crackers, chips, corndogs, peanut butter and jelly sandwiches, goldfish, banana, grapes, fruit snacks, oreos, and poptarts. Julian received $89 \%, 189 \%$, and $43 \%$ of his daily caloric, protein, and fluid needs respectively. He was in the $67^{\text {th }}, 72^{\text {nd }}$, and $68^{\text {th }}$ percentile for height, weight, body-mass index, respectively. Our program's registered dietician estimated that Julian's intake was deficient in calcium, chromium, magnesium, manganese, potassium, zinc, and vitamins $\mathrm{B} 5, \mathrm{~B} 7, \mathrm{D}$, and $\mathrm{E}$.

\section{Setting and Materials}

Feeders conducted control and mitigation sessions with John, Maisy, Hope, Emilia, Diego, Jade, and Julian in 4-m by 4-m rooms in a pediatric feeding disorders clinic. Session rooms contained a rectangular table, utensils, a food tray, a scale, a chair for the feeder, weightappropriate seating for the child; colored bowls for Emilia, Diego, and Julian; colored tablecloths for Emilia and Julian; and 10-cm by 17-cm colored cards for Diego and 91-cm by 122-cm colored cards for Julian. The session rooms had adjacent observation rooms with one-way observation and two-way audio and sound. Caregivers conducted Jade's sessions in their home and therapists 
observed Jade's home sessions using Vidyo, a secure video-conferencing software. John and Emilia sat in a Special Tomato Soft-Touch sitter that we secured to a regular kitchen chair and Hope sat in a regular kitchen chair. Maisy and Julian sat in a booster seat that we secured to a regular kitchen chair. Diego and Jade sat in a high chair.

Caregivers selected 16 foods that the child currently did not consume from a list provided by a therapist to target in treatment with solid foods. Caregivers of each participant selected four fruits, four proteins, four starches, and four vegetables with input from our program's dietician to promote appropriate macro and micronutrient intake. Caregivers of John, Maisy, Hope, and Jade also selected an age, calorically, and nutritionally appropriate liquid as increasing liquid consumption was a target of treatment for these participants, but not for the other participants.

\section{Feeders}

Feeders were the child's biological mother (Maisy, Hope, Emilia, Jade, and Diego) and father (John and Hope) and trained clinic therapists. Therapists also served as observers. Therapists were permanent employees with a Bachelor's or Master's Degree in psychology, applied behavior analysis (ABA), or a related field; predoctoral interns in an American Psychological Association-approved predoctoral internship program; students in an ABA doctoral program; practicum students in an undergraduate psychology program or related field; or a combination of these individuals. Training for therapists included didactic modules with posttests, competency training for session and food preparation, and in-vivo training to competency for child assessment and treatment and caregiver-training procedures.

\section{Dependent Variables, Procedural Integrity, and Reliability}

Observers used BDataPro ${ }^{\circledR}$, a specialized data-collection program developed in our clinic, to collect data on inappropriate mealtime behavior, acceptance, and procedural integrity in the control and mitigation conditions (Bullock et al., 2017). During caregiver training, observers 
scored occurrences and nonoccurrences of procedural integrity on each intervention component using an excel spreadsheet that generated a graph from the inputted data.

\section{Dependent Variables}

Observers scored the frequency of inappropriate mealtime behavior when the spoon or cup was within arm's reach of the child and each time the child (a) moved the middle of his or her mouth $45^{\circ}$ or more in any direction or $6.4 \mathrm{~cm}$ in any direction away from the utensil; (b) changed direction or paused for $1 \mathrm{~s}$ and then moved the head another $45^{\circ}$ in any direction or $6.4 \mathrm{~cm}$ in any direction relative the previous position; or if the child's hand, arm, or anything in the child's hand (e.g., bib) touched the spoon, cup, bite, drink or any part of the feeder's hand or arm from the elbow down that the feeder was using to present, deposit, and re-present the bite or drink; (c) threw the spoon or cup; (d) placed his or her hand, arm, toy, or bib within $5 \mathrm{~cm}$ of the mouth; and (e) moved more than $2.5 \mathrm{~cm}$ from and was no longer touching the spoon, cup, feeder's hand or arm from the elbow down, or the child's own mouth and then returned to the spoon, cup, feeder's hand or arm from the elbow down, or the child's own mouth. A presentation occurred the first time the feeder touched the utensil to the child's lips to offer the child the opportunity to accept that bite or drink initially. Observers scored the occurrence of acceptance when the child (a) opened his or her mouth in the absence of crying, screaming, yelling, or refusal statements and the entire bite or drink passed the plane of the wet vermillion of the child's lips within $5 \mathrm{~s}$ of the initial presentation; or (b) the child opened his or her mouth and leaned forward while engaging in negative vocalizations and the entire bite or drink passed the plane of the wet vermillion of the child's lips within $5 \mathrm{~s}$ of the initial presentation. The number of opportunities for observers to score acceptance corresponded to the number of bites or drinks the feeder presented. We defined an entire bite or drink as the volume of solids or liquids the feeder presented on the utensil minus a pea-sized volume or smaller. Observers scored the occurrence of mouth clean when there was no food or liquid in the mouth before or at the initial mouth-clean check, which occurred $30 \mathrm{~s}$ 
after the feeder deposited the bite or drink into the child's mouth. Observers did not score mouth clean if the absence of food or liquid in the mouth was due to expulsion. We defined expulsion as any food or liquid larger than a pea-size volume that passed the plane of the wet vermillion of the child's lips after the bite or drink had entered the child's mouth. The number of opportunities for observers to score mouth clean corresponded to the number of bites or drinks that entered the child's mouth initially, not including bites or drinks that entered the mouth during re-presentation.

BDataPro ${ }^{\circledR}$ converted the frequency of inappropriate mealtime behavior to responses per minute by dividing the number of inappropriate mealtime behaviors during the session by the duration the utensil was within arm's reach of the child. BDataPro ${ }^{\circledR}$ converted acceptance to a percentage after dividing the number of acceptances by the total number of presentations.

BDataPro ${ }^{\circledR}$ converted mouth clean to a percentage after dividing the number of mouth cleans by the total number of bites or drinks that entered the mouth.

\section{Procedural Integrity}

Correct utensil placement assessed procedural integrity for components specific to the feeder's utensil use. Observers scored duration of correct-utensil placement in the control and mitigation conditions by activating a duration key on BDataPro ${ }^{\circledR}$ when the feeder met the criterion for correct-utensil placement and deactivating the duration key when the feeder did not

meet the criterion for correct-utensil placement for $3 \mathrm{~s}$ or more. Across all conditions of the study, observers scored correct-utensil placement when the feeder presented the utensil to the child's lips at the scheduled interval; removed the utensil after the bite or drink entered the mouth; and held the utensil to the side of the child's mouth if the child coughed, gagged, or vomited while the feeder was holding the utensil at the child's lips. Observers scored correct-utensil placement during the function-based baseline of the control and mitigation conditions if the feeder removed the utensil after the occurrence of inappropriate mealtime behavior. Observers scored correctutensil placement during function-based extinction of the control and mitigation conditions when 
the feeder held the spoon to the child's lips if the child did not meet criterion for acceptance until he or she could deposit the bite or drink into the mouth or the time-cap elapsed and used the utensil to re-present the expelled bite or drink into the mouth within $3 \mathrm{~s}$ of expulsion. BDataPro® converted duration of correct-utensil placement to a percentage after dividing the duration of correct-utensil placement by the total session time. Mean correct-utensil placement during the function-based reinforcement in Context A was 99\% (range, 99\% to 100\%) for caregivers and $99 \%$ (range, $99 \%$ to $100 \%$ ) for therapists during the control condition and $99 \%$ (range, $91 \%$ to $100 \%$ ) for caregivers and $99 \%$ (range, $99 \%$ to $100 \%$ ) for therapists during the mitigation condition. Mean correct utensil placement during function-based extinction in Context B was $99 \%$ (range, $88 \%$ to $100 \%$ ) for therapists during the control condition and $99 \%$ (range, $88 \%$ to $100 \%$ ) for caregivers and $99 \%$ (range, $89 \%$ to $100 \%$ ) for therapists during the mitigation condition. Mean correct utensil placement during function-based extinction in Context A was $99 \%(98 \%$ to $100 \%$ ) for caregivers and $99 \%$ (range, $99 \%$ to $88 \%$ ) for therapists during the control condition and $99 \%$ (range, $99 \%$ to $100 \%$ ) for caregivers and $99 \%$ (range, $99 \%$ to $100 \%$ ) for therapists during the mitigation condition.

Correct procedure assessed procedural integrity for components other than those specific to the feeder's utensil use. Observers only scored correct procedure if the feeder completed every component described below. Observers did not score correct procedure if the feeder did not perform a component or performed a component incorrectly. Observers scored the occurrence of correct procedure in the control and mitigation conditions after the mouth-clean check or at the end of the presentation interval if the feeder (a) used the correct utensil and presented the specified bolus size (e.g., 2 cc); (b) delivered a vocal prompt to "Take a bite (drink)" while touching the utensil to the child's lips within $5 \mathrm{~s}$ of the scheduled presentation; (c) deposited the bite or drink within $5 \mathrm{~s}$ of presentation if the child met the criterion for acceptance; (d) delivered vocal, behavior-specific praise (e.g., "Great job taking your drink") within $5 \mathrm{~s}$ of acceptance and 
mouth clean; (e) conducted a mouth-clean check approximately $30 \mathrm{~s}$ after the feeder deposited the bite or drink into the child's mouth; (f) delivered the vocal prompt to "Swallow your bite (drink)" within $5 \mathrm{~s}$ of the mouth-clean check if the child packed (i.e., held food or liquid larger than a peasize volume in the mouth) and delivered the swallow prompt every $30 \mathrm{~s}$ if the child packed on the fifth bite or drink presentation until there was no food or liquid in the child's mouth or until 10 min elapsed; (g) provided no differential consequences for coughing, gagging, or vomiting; and (h) presented the next bite or drink within $5 \mathrm{~s}$ of the next scheduled presentation interval.

Observers scored correct procedure for caregivers and therapists separately when therapists fed and caregivers delivered other components of the intervention protocol (i.e., consequences for acceptance and mouth clean and prompts at the bite presentation and mouth-clean check) during the mitigation condition. DataPro ${ }^{\circledR}$ converted correct procedure to a percentage after dividing the number of bite or drink presentations with correct procedure by the total number of bite or drink presentations. Mean correct procedure during the function-based reinforcement in Context A was $98 \%$ (range, $80 \%$ to $100 \%$ ) for caregivers and therapists during the control condition and $97 \%$ (range $80 \%$ to $100 \%$ ) for caregivers and $99 \%$ (range $80 \%$ to $100 \%$ ) for therapists during the mitigation condition. Mean correct procedure during function-based extinction in Context B was $100 \%$ for therapists during the control condition and $98 \%$ (range, $80 \%$ to $100 \%$ ) for caregivers and $100 \%$ for therapists during the mitigation condition. Mean correct procedure during functionbased extinction in Context A was $99 \%$ (range, $80 \%$ to 100\%) for caregivers and $100 \%$ for therapists during the control condition and $97 \%$ (range, $80 \%$ to $100 \%$ ) for caregivers and $100 \%$ for therapists during the mitigation condition.

Observers scored occurrences of incorrect attention once during each presentation interval in the control and mitigation conditions when the feeder (a) did not provide behaviorspecific praise within $5 \mathrm{~s}$ of acceptance and mouth clean, (b) delivered behavior-specific praise if the bite or drink entered the child's mouth after $5 \mathrm{~s}$ of presentation, or (c) delivered behavior- 
specific praise if there was food or liquid in the child's mouth at the mouth-clean check. Observers also scored incorrect attention during escape baseline and function-based extinction when the feeder delivered attention (e.g., coaxes, reprimands) within $5 \mathrm{~s}$ of inappropriate mealtime behavior. Observers scored incorrect attention during the escape and attention baseline when the feeder did not deliver attention within $5 \mathrm{~s}$ of inappropriate mealtime behavior. BDataPro ${ }^{\circledR}$ converted incorrect attention to a percentage after dividing occurrences of incorrect attention by the total number of bite presentations in the session. Mean incorrect attention during function-based reinforcement in Context $\mathrm{A}$ was $1 \%$ (range, $0 \%$ to $20 \%$ ) for caregivers and $0 \%$ for therapists during the control condition and $0.3 \%$ (range, $0 \%$ to $20 \%$ ) for caregivers and $0 \%$ for therapists during the mitigation condition. Mean incorrect attention during function-based extinction in Context B was $0 \%$ for therapists during the control condition and $0.6 \%$ (range, $0 \%$ to $20 \%$ ) for caregivers and $0 \%$ for therapists during the mitigation condition. Mean incorrect attention during the renewal test was $0.5 \%$ (range, $0 \%$ to $20 \%$ ) for caregivers and $0 \%$ for therapists during the control condition and $1 \%(0 \%$ to $20 \%)$ for caregivers and $0 \%$ for therapists during the mitigation condition.

\section{Interobserver Agreement}

Two observers simultaneously and independently collected data on a mean of $54 \%$ and $66 \%$ of sessions in the control and mitigation condition, respectively. We trained observers to collect data on BDataPro ${ }^{\circledR}$ and using a streamline excel graph with $80 \%$ or higher interobserver agreement for three consecutive sessions.

The BDataPro ${ }^{\circledR}$ software calculated interobserver agreement by dividing each session into 10-s intervals. BDataPro ${ }^{\circledR}$ calculated exact agreement coefficients for inappropriate mealtime behavior by dividing the number of exact agreements (observers scored the same frequency of the behavior in an interval) by the number of exact agreements plus disagreements (observers scored different frequencies of the behavior in an interval) and converting this ratio to 
a percentage. Mean interobserver agreement was $94 \%$ (range, $73 \%$ to $100 \%$ ) and $96 \%$ (range, $71 \%$ to $100 \%$ ) for inappropriate mealtime behavior during the control and mitigation condition, respectively. BDataPro ${ }^{\circledR}$ calculated total agreement coefficients for acceptance, mouth clean, correct utensil placement, correct procedure, and incorrect attention by dividing the total number of agreements (i.e., both observers scored the occurrence or nonoccurrence of the behavior in the interval) by the total number of agreements plus disagreements (one observer scored and the other observer did not score the occurrence of the behavior in the interval) and converting this ratio to a percentage. Mean interobserver agreement during the control condition was $99 \%$ (range, $80 \%$ to $100 \%$ ) for acceptance, $99 \%$ (range, $80 \%$ to $100 \%$ ) for mouth clean, $99 \%$ (range, $90 \%$ to $100 \%$ ) for correct utensil placement, $99 \%$ (range, $80 \%$ to 100\%) for correct procedure, and $99 \%$ (range, $80 \%$ to $100 \%$ ) for incorrect attention. Mean interobserver agreement during the mitigation condition was $98 \%$ (range, $80 \%$ to $100 \%$ ) for acceptance, $99 \%$ (range, $80 \%$ to $100 \%$ ) for mouth clean, $99 \%$ (range, $85 \%$ to $100 \%$ ) for correct utensil placement, $98 \%$ (range, $80 \%$ to $100 \%$ ) for correct procedure, and $99 \%$ (range, $80 \%$ to $100 \%$ ) for incorrect attention.

\section{Experimental Design}

\section{Functional Analysis}

We used a pairwise design (Bachmeyer et al., 2009) in the functional analysis to identify environmental variables maintaining inappropriate mealtime behavior by comparing levels of inappropriate mealtime behavior in the escape and attention test conditions to the control condition.

\section{Control and Mitigation Conditions}

We used a three-phase ABA arrangement to evaluate the control and mitigation conditions. During Context A, the caregiver or therapist delivered function-based reinforcement for inappropriate mealtime behavior during the control and mitigation conditions. During Context 
$\mathrm{B}$, the therapist implemented function-based extinction of inappropriate mealtime behavior during the control and mitigation conditions. The therapist also used fading to introduce the caregiver as the feeder for John, Maisy, Hope, Emilia, Diego, and Jade, and new foods for Diego in the mitigation condition. During the return to Context A, the caregiver or therapist implemented function-based extinction of inappropriate mealtime behavior.

\section{General Procedure}

Feeders conducted five 40-min meals per day with at least $40 \mathrm{~min}$ between the end of one meal and the start of the next meal. For example, Jade's meal schedule was 9:00 a.m. to 9:40 a.m., 10:20 a.m. to 11:00 a.m., 11:40 a.m. to 12:20 p.m., 2:00 p.m. to 2:40 p.m., 3:20 p.m. to 4:00 p.m. for Meals 1 to 5, respectively. Each session generally consisted of five bite or drink presentations with approximately 1 min between each session during which feeders and observers prepared for the next session (e.g., weighed foods, recorded gram intake, reset data-collection program). The number of sessions per meal depended on the duration of each session within the meal, which depended on the child's behavior. Mean number of sessions per meal was four for Maisy; five for John, Hope, Emilia, Diego, and Julian; and six for Jade. The feeder generally conducted control sessions in some meals and mitigation sessions in other meals. The feeder conducted solids sessions in all meals for Emilia, Diego, and Julian. The feeder generally conducted solids sessions in some meals and liquids sessions in other meals for John, Maisy, Hope, and Jade. The only time the feeder conducted the control and mitigation conditions in the same meal is if the child met the criterion for us to change phases and implement the contingencies in the next context, like a change from Context A to Context B, and we needed to equate the number of sessions between conditions before changing phases. For example, if the child met criterion in solids sessions during Meal 1 in Context A to change to Context B, then the feeder stopped conducting solids sessions in Meal 1 in Context A so that we could transition to solids sessions in Context B in Meal 2. If 20 min or more remained in Meal 1 and the child had 
not met criterion to change to Context $\mathrm{A}$ in liquids sessions, then the feeder conducted liquids sessions in Context A for the remainder of Meal 1.

Feeders presented $1 \mathrm{cc}$ of pureed food, which is table food blended until smooth with liquid added as needed, on a small maroon spoon in solids sessions. The feeder presented four of the 16 caregiver-selected foods, one fruit, one protein, one starch, and one vegetable, in the functional analysis; eight of the 16 caregiver-selected foods, two fruits, two proteins, two starches, and two vegetables, during the control and mitigation conditions for John, Maisy, Hope, Emilia, Jade, and Julian; 16 caregiver-selected foods during the control and mitigation conditions for Diego. The feeder presented the same foods in every condition in every phase to control for potential differences in children's behavior as a function of food type (Patel, Piazza, Santana, \& Volkert, 2002). The feeder randomly selected the order of food presentation before the start of the session.

The feeder presented the caregiver-selected formula for John, Maisy, Hope, and Jade for liquid sessions in all conditions. Feeders presented $2 \mathrm{cc}$ of Vanilla Pediasure 1.0 with and without fiber in a small pink cut-out cup for Jade and John, respectively. Feeders presented 4 cc of Vanilla Puramino Junior and Peptamin Junior 1.5 in a pink cut-out cup for Maisy and Hope, respectively.

During each session, the feeder presented a bite or drink by touching the utensil to the child's lips while saying, "Take a bite (drink)." The feeder deposited the bite or drink if the child met the criterion for acceptance and delivered behavior-specific praise for acceptance (e.g., “Good job taking your bite!”). The feeder conducted a mouth-clean check approximately $30 \mathrm{~s}$ after the feeder deposited the bite or drink into the child's mouth by saying, "Show me, ahh." If the child did not open his or her mouth within $3 \mathrm{~s}$ of the verbal and model prompt, the feeder inserted a small rubber-coated spoon between the child's lips and turned the spoon $90^{\circ}$ to prompt the child to open his or her mouth. The feeder delivered behavior-specific praise (e.g., "Good job 
swallowing your bite!") for mouth clean or a verbal prompt to "Swallow your bite (drink)" if the child was packing and presented the next bite or drink. If the child showed the feeder a mouth clean before 30 s elapsed to signal the mouth-clean check, the feeder delivered behavior-specific praise and waited until the mouth-clean interval elapsed before presenting the next bite or drink. If the child had packed food or liquid in his or her mouth after the fifth bite or drink presentation, the feeder conducted a mouth check and delivered the verbal prompt to swallow every $30 \mathrm{~s}$ until no food or liquid was in the child's mouth or until $10 \mathrm{~min}$ had elapsed from the start of the session. The feeder delivered no differential consequence for coughing, gagging, or vomiting. The feeder moved the utensil to the side of the child's mouth if the child coughed, gagged, or vomited while the utensil was at the child's lips and moved the utensil back to the child's lips when the child stopped coughing, gagging, or vomiting.

\section{Functional Analysis}

We conducted unstructured and structured direct observations of the caregiver feeding his or her child before conducting a functional analysis (Vaz \& Piazza, 2010). During the unstructured format, the caregiver presented preferred and nonpreferred foods separately to his or her child for 10 min each. Next, we conducted five structured observations in which the caregiver (a) attempted to feed the child pureed bites on a spoon, (b) attempted to feed the child drinks in a cup, (c) presented pureed bites on a spoon and vocally prompted the child to self-feed, (d) presented table-textured bites on a spoon and vocally prompted the child to self-feed, and (d) presented drinks in a cup and vocally prompted the child to self-feed. We used information from these direct observations to inform which test conditions we included in the functional analysis. Direct observations indicated that caregivers delivered escape and attention following inappropriate mealtime behavior; thus, we conducted escape and attention conditions with each participant. Feeders were the caregiver for Maisy and Diego, therapist for Julian, and the caregiver and therapist for John, Hope, Emilia, and Jade. Feeders conducted functional analyses 
for solids and liquids with John, Maisy, Hope, and Jade, and solids only with Emilia, Diego, and Julian because only solids were a treatment target. Caregivers conducted the functional analysis before the therapist for solids with John and Emilia and after the therapist for liquids for John and solids and liquids for Hope and Jade.

\section{Control and Mitigation Conditions}

One purpose of the study was to assess whether renewal of inappropriate mealtime behavior occurred when we implemented function-based-extinction treatment for children with feeding disorders. The context changes were (a) replacing the therapist as feeder in the clinic with a caregiver as feeder in the clinic for John, Maisy, Hope, and Emilia, (b) replacing the therapist feeding one set of four foods in the clinic with the caregiver feeding a different set of four foods in the clinic for Diego, (c) replacing the therapist as feeder in the clinic with a caregiver as feeder at home for Jade, and (d) replacing the setting in the clinic with a simulated home environment for Julian. A second purpose was to assess whether our renewal-mitigation procedures would mitigate or prevent renewal in a condition that was like the one in which we tested for renewal with each participant in the control condition. The arrangement for the mitigation condition was like the control condition except that we implemented our renewal-mitigation procedures during extinction in Context B. We selected the treatment targets for the control and mitigation conditions based on the child's clinical goals. Thus, we assessed renewal effects during liquids and solids treatment for John, Maisy, Hope, and Jade because solids and liquids were their treatment targets. We assessed renewal effects during solids treatment with two different sets of foods for Emilia, Diego, and Julian because only solids were a treatment target. We used colored stimuli to aid Emilia's, Diego's, and Julian's discrimination of the conditions because the feeder

presented pureed food in both conditions. Pureed foods are difficult to discriminate because they do not have as many distinctive properties as the same foods at table texture. We also randomly chose the order in which we conducted sessions of function-based extinction in the renewal test 
across the control and mitigation conditions with each participant. We conducted renewal test sessions in the control condition first and the mitigation condition second with John, Maisy, Emilia, and Jade. We conducted renewal test sessions in the mitigation condition first and the control condition second with Hope, Diego, and Julian.

We randomly assigned solids for John and Hope and liquids for Maisy and Jade to the control condition and liquids for John and Hope and solids for Maisy and Jade to the mitigation condition. We randomly assigned four foods, one fruit, one protein, one starch, and one vegetable, to a food set for Emilia, Diego, and Julian. For Emilia, we randomly assigned Set 1 foods (baked bean, green bean, pancake, and pear), orange bowls, and an orange table cloth to the control condition and Set 2 foods (banana, carrot, chicken, and potato), blue bowls, and a blue table cloth to the mitigation condition. For Diego, we randomly assigned blue bowls, and a blue $10-\mathrm{cm} \times 17-\mathrm{cm}$ card to the control condition and orange bowls, and an orange 10-cm x $17-\mathrm{cm}$ card to the mitigation condition. We also randomly assigned one food set to each context of the control and mitigation conditions. We randomly assigned Set 1 foods to Context A and Set 2 foods to Context B of the control condition and Set 3 foods to Context A and Set 4 foods to Context B of the mitigation condition. Set 1 was banana, cauliflower, egg, and pancake; Set 2 was applesauce, green bean, hamburger, and oatmeal; Set 3 was broccoli, sweet potato, and tuna; and Set 4 was chicken, potato, strawberry, and squash. For Julian, we randomly assigned Set 1 foods (chicken, potato, applesauce, and carrot), orange bowls, orange table cloth, and an orange 91-cm by $122-\mathrm{cm}$ card to the control condition and Set 2 foods (green bean, baked bean, wheat bread, and pear), blue bowls, blue table cloth, and a blue $91-\mathrm{cm}$ by $122-\mathrm{cm}$ card to the mitigation condition.

The feeder started each corresponding phase of the control and mitigation conditions on the same day. The feeder always transitioned from Context A to Context B on the same day and transitioned from Context B back to Context A on the same day. For example, if the feeder 
conducted the last session of Context B in the control condition on Tuesday, then he or she would conduct the first session of Context A in the control condition on that same Tuesday. If the feeder transitioned from Context B to Context A in the control condition on Tuesday, then he or she would conduct the transition from Context $\mathrm{B}$ to Context $\mathrm{A}$ in the mitigation condition on the same Tuesday. The feeder followed the general procedure described above and the specific procedure described below for control and mitigation conditions. The feeder conducted the same number of sessions for each Context in the control and mitigation conditions. We equated the number of sessions in each context across the control and mitigation conditions before changing from context to context to ensure that our manipulations, rather than unequal exposure to the experimental conditions, produced the observed effects. For example, if the number of sessions for Context A was 10 in the control condition, then the number of sessions for Context $\mathrm{A}$ in the mitigation condition was 10 .

\section{Context A Reinforcement (Control and Mitigation Conditions)}

The caregiver delivered functional reinforcement following inappropriate mealtime behavior in the clinic for John, Maisy, Hope, Emilia, and Diego and the home for Jade. The therapist delivered functional reinforcement following inappropriate mealtime behavior in a simulated home for Julian. The functional reinforcer was escape and attention for John, Maisy, Hope, Emilia, and Diego; and escape for Julian. The functional reinforcer for Jade was escape and attention during solids and escape during liquids. In addition, the caregiver presented Set 1 foods in the control condition and Set 3 foods in the mitigation condition for Diego. Therapists and observers were in the adjacent observation room for John, Maisy, Hope, Emilia, and Diego; clinic observing via Telehealth for Jade; and simulated home for Julian.

\section{Context B Extinction (Control Condition)}


A therapist conducted sessions with the child in a session room. The therapist implemented escape extinction and attention extinction for John, Maisy, Hope, Emilia, and Diego. The therapist implemented escape extinction for Julian. The therapist implemented escape extinction and attention extinction during solids and escape extinction during liquids for Jade. The escape extinction component consisted of the feeder keeping the utensil at the child's lips until the child met the criterion for acceptance, until the child opened his or her mouth such that the feeder could deposit the bite or drink into the mouth if the child did not meet the criterion for acceptance, or until the 10-min time-cap elapsed. If the child expelled the bite or drink, the feeder used the utensil to scoop the expelled bite or drink and re-present it into the child's mouth. If the child was expelling the bite or drink during the mouth-clean check, the feeder re-presented the expelled bite or drink until the child kept the bolus in his or her mouth for $3 \mathrm{~s}$, then the feeder presented the next bite or drink. If the child expelled the bite or drink during the mouth-clean check on the fifth bite or drink presentation, the feeder re-presented the bite or drink until the child met the criterion for a mouth clean or until 10 min elapsed from the start of the session after which the feeder started the next session. If the child was packing at the mouth-clean check, the feeder said, "Swallow your bite (drink)," and presented the next bite or drink. If the child was packing solids or liquids on the fifth mouth-clean check, the feeder prompted the child to swallow every $30 \mathrm{~s}$ until the child swallowed the bolus or until 10 min elapsed from the start of the session. The feeder wiped out packed food or liquid from the child's mouth after the time cap elapsed. During the attention-extinction component, the feeder provided no differential consequence for inappropriate mealtime behavior. The therapist presented Set 2 foods for Diego.

Caregiver Training (Control Condition). Therapists trained caregivers to implement the function-based extinction protocol before we transitioned from therapist-fed sessions in Context B to caregiver-fed sessions in Context A. First, caregivers were in the observation room and watched the last three sessions of Context B in which the therapist fed the child using the 
function-based extinction protocol. We used visual inspection to determine when the last three sessions of Context B would occur by evaluating the number of sessions required for the child to meet the criteria to change to Context $\mathrm{A}$ in the control and mitigation conditions. If the child met the criteria to change from Context B to Context $\mathrm{A}$ in the mitigation condition first, we used the number of sessions from Context B of the mitigation sessions to determine when the last three sessions would occur in Context B of the control condition. Second, therapists provided caregivers with a written protocol and explained the purpose of function-based extinction. After caregivers read the protocol, the therapist answered the caregiver's questions. Third, therapists trained the caregiver in a session room without the child present. The training method included the therapist narrating and modeling a protocol component followed by the caregiver role-playing the modeled protocol component. This pattern of therapist narrate and model - caregiver role play with confederate child continued until the therapist had narrated and modeled and the caregiver had role-played every component of the protocol. In the final step, the therapist narrated and modeled the entire protocol followed by the caregiver role-playing the entire protocol.

The therapist narrated and modeled and the caregiver role-played the protocol components with another therapist who served as a confederate child. Confederate children followed a script that dictated child behavior on each trial and ensured that the confederate child emitted every possible behavior the caregiver would encounter when he or she fed his or her child. We did this so the caregiver could observe and practice responses to each possible child behavior that he or she would encounter when he or she fed his or her child. We also used direct observations of the child's behavior in the functional analysis and function-based baseline to program the frequency of the scripted confederate-child behaviors during role-play. For example, if the caregiver's child frequently coughed and gagged, the script programmed the confederate child to cough and gag on multiple trials so the caregiver received additional practice responding to behavior specific to his or her child. 
The order of trained and practiced components was (a) consequences for acceptance and mouth clean, (b) prompts at the bite- or drink-presentation interval and the mouth-clean check, and (c) the entire protocol. The protocol consequences included behavior-specific praise for acceptance, no praise if the child did not meet the criterion for acceptance, behavior-specific praise at the mouth clean check if the child met the criterion for a mouth clean, and no praise if the child did not meet the criterion for mouth clean. The protocol prompts were saying, "Take a bite" when the utensil touched the child's lips; saying, "Show me, ahh," at the mouth-clean check, and saying, "Swallow your bite," if the child did not meet the criterion for a mouth clean. The entire protocol included presenting the utensil to the child's lips; depositing the bite or drink if the child met the criterion for acceptance; holding the utensil at the child's lips until the child opened his or her mouth such that the caregiver could deposit the bite or drink if the child did not meet the criterion for acceptance; re-presenting expelled bites or drinks; holding the utensil to the side of the lips if the child was coughing, gagging, or vomiting while the utensil was at the child's lips but providing no additional differential consequences for coughing, gagging, or vomiting; and providing no differential consequence for inappropriate mealtime behavior. We used an additive approach during caregiver role plays with the confederate child. The caregiver implemented the intervention components he or she previously mastered during trials with the confederate child when we were teaching the caregiver a new intervention component. For example, after the therapist trained the caregiver to deliver consequences, the caregiver continued to deliver consequences to the confederate child while he or she was learning to deliver the prompts. Therapists provided behavior-specific praise for correct protocol implementation and delivered corrective feedback for incorrect protocol implementation during and after each trial. The mastery criterion for role play was that the caregiver implemented the protocol with $80 \%$ or higher integrity for one five-bite or drink session for consequences for acceptance (e.g., "Good job taking your bite") and mouth clean ("Good job swallowing your bite"), prompts at the bite or drink presentation (e.g., "Take a bite") and at the mouth-clean check (i.e., "Show me, ahh," and 
"Swallow your bite or drink"); and three consecutive five-bite or drink sessions for the entire protocol. After the caregiver met the role-play criterion while implementing the entire protocol with the confederate child, the caregiver implemented the protocol with his or her child during the renewal test in Context A (see below).

\section{Context B Extinction (Mitigation Condition)}

A therapist fed the child and implemented function-based extinction as described for the control condition above. The therapist and observer were in the session room during caregiver training and during sessions in the mitigation condition. In addition, we used the procedure described below to train the caregiver to implement the function-based extinction protocol and to transition from the therapist to the caregiver as feeder for John, Maisy, Hope, Emilia, Diego, and Jade and introduce new foods for Diego. The caregiver observed the first three sessions of function-based extinction from the adjacent observation room and reviewed the protocol with a therapist before we started fading the caregiver into the session room.

\section{Caregiver Training and Mitigation Procedure (Mitigation Condition). Abruptly}

changing from the therapist to the caregiver as feeder or adding new foods is likely a discriminable context change that has occasioned renewal of inappropriate mealtime behavior in previous studies (Ibañez et al., 2019; Kelley et al., 2018). The purpose of the renewal-mitigation procedure was to change the context incrementally. We wanted the change from therapist to caregiver and the addition of new foods to be less discriminable to the child during the mitigation condition to prevent the renewal of inappropriate mealtime behavior during this condition.

We used a training procedure like that described for the control condition. The difference was that after the caregiver demonstrated mastery of a trained component of the protocol with the confederate child, the therapist and caregiver then conducted a session with the caregiver's child. During that session with the caregiver's child, the therapist fed the child and the caregiver 
implemented the function-based extinction component(s) for which he or she had demonstrated mastery with the confederate child. For example, after the therapist modeled delivering consequences, the caregiver role played delivering consequences with the confederate child. After the caregiver met mastery criterion for delivering consequences during role play with the confederate child, the caregiver delivered consequences to his or her child while the therapist fed the child using the function-based extinction protocol. The therapist and caregiver conducted sessions with the caregiver's child until the caregiver demonstrated mastery of the target protocol component during those sessions with his or her child.

The therapist and caregiver then repeated the sequence of therapist narrate and model, caregiver role play with confederate child, therapist and caregiver implement protocol with caregiver's child until the caregiver had demonstrated mastery implementing the entire protocol with his or her child with the therapist sitting next to the caregiver in the room. The mastery criterion for role-play was $80 \%$ or higher correct utensil placement and correct procedure and $20 \%$ or lower incorrect attention for one five-bite or drink session for consequences for acceptance and mouth clean and prompts and three consecutive five-bite or drink sessions for the entire protocol.

After the caregiver implemented the function-based extinction procedure, the therapist and observer gradually faded his or her position until he or she was out of the room. The mastery criterion for therapist position fading was $80 \%$ or higher acceptance, correct utensil placement, and correct procedure; $20 \%$ or lower incorrect attention; and zero instances of inappropriate mealtime behavior for three consecutive five-bite or drink sessions. The therapist moved from sitting next to the caregiver, to across the table, then next to the door of the session room. The observer moved out of the room at the same time the therapist moved across the table. The therapist provided immediate corrective feedback for most caregiver errors. Occasionally, the therapist provided corrective feedback at the end of the session. For example, if the caregiver did 
not present all four foods during a session, the therapist reminded the caregiver at the end of the session to present all four foods during each five-bite or session. Therapists provided frequent positive feedback statements while sitting next to the caregiver. Therapists used an informal method of fading the number of positive feedback statements as they faded out of the room. Therapists generally based the number of praise statements on caregivers' procedural integrity from the previous sessions. For instance, if the caregiver implemented the protocol with high integrity in several sessions, the therapist delivered fewer praise statements in the next session. If the caregiver made many procedural errors in one session, the therapist increased the number of praise statements in the next session so that he or she delivered praise and corrective feedback.

For Diego, we used the same caregiver-fading protocol described above in addition to fading in Set 3 foods his mother presented in Context A of the mitigation condition into Context B of the mitigation condition. We randomly selected to fade Diego's mother in as feeder before fading in Set 3 foods. The trained therapist only presented Set 4 foods during caregiver fading. During caregiver fading, Diego's mother presented Set 4 foods until she implemented the function-based extinction protocol with $80 \%$ or higher correct utensil placement, correct procedure, and $20 \%$ or lower incorrect attention for three consecutive five-bite sessions. Next, we started food fading. Diego's mother was the feeder during food fading, and we faded in one Set 3 food at a time. Diego's mother always presented one Set 3 food with three Set 4 foods until she had introduced all Set 3 foods after which we faded the ratio of Set 3 foods to Set 4 foods. Diego's mother always presented one food from each of the groups of fruit, protein, starch, and vegetable. The criterion for introducing a Set 3 food was $80 \%$ or higher caregiver correct utensil placement and correct procedure and $20 \%$ or lower incorrect attention for three consecutive fivebite sessions. We used integrity as the criterion for adding in foods initially to model a similar fading progression as we used in the caregiver-fading protocol. We also randomized the bite number in which Diego's mother introduced each Set 3 food to control for order effects. For 
example, Diego's mother introduced tuna on Bite 3 of one five-bite session and avocado on Bite 5 of a different five-bite session. After Diego's mother introduced a Set 3 food during one fivebite session, she presented the Set 3 food and the same three Set 4 foods in random order during two more five-bite sessions. Next, she presented two Set 3 foods and two Set 4 foods for one fivebite session, then three Set 3 foods and one Set 4 food for three consecutive five-bite sessions. The criterion for fading the ratio of Set 3 to Set 4 foods was $80 \%$ or higher acceptance, correct utensil placement, correct procedure; $20 \%$ or lower incorrect attention; and zero instances of inappropriate mealtime behavior.

For Jade, we used the same systematic fading protocol described above to fade in Jade's mother as the feeder, and we arranged the session room to look like her kitchen at home. Research suggests that increasing the similarity between contexts is a method that mitigates renewal (Bandarian-Balooch \& Neumann, 2011; Podlesnik et al., 2017; Todd et al., 2012). Therefore, we used context similarity for Jade and Julian by arranging the session room (i.e., Context B) similar to the home or simulated home environment (i.e., Context A) to mitigate renewal following a setting change during extinction. For Jade, we projected a picture of the view of the kitchen that Jade would see when she sat in her highchair at home onto the wall of the session room and placed common items from her home like toys, a plant, lotion, and a table centerpiece in the session room. For Julian, we increased the similarity between the clinic room and a simulated home environment by projecting a picture of the view of the simulated home that Julian would see when he sat in his booster seat onto the wall of the session room and placed common items from the simulated home like a rug, lamps, paintings, and plants in the session room.

\section{Context A Extinction (Renewal Test, Control and Mitigation Conditions)}

The contingencies and contextual arrangements during the renewal tests in Context A were identical in the control and mitigation conditions. The caregivers or therapists (Julian) 
implemented function-based extinction as described for the Context B control condition in the clinic with John, Maisy, Hope, Emilia, Diego, and Julian and in the home with Jade. Diego's caregiver presented Set 1 foods during the control condition and Set 3 foods during the mitigation condition. We conducted at least five consecutive five-bite or drink sessions of the renewal test in the control and mitigation conditions across participants. Therapists and observers were not in the session room when the caregiver delivered function-based extinction during Context A for John, Maisy, Hope, Emilia, and Diego; they observed sessions in the observation room. Therapists delivered corrective feedback as necessary over the two-way audio system from the adjacent observation room for John, Maisy, Hope, Emilia, and Diego and over Vidyo for Jade to ensure caregivers maintained high levels of integrity.

\section{Social Validity}

We gave caregivers a 5-item questionnaire and asked them to rate the acceptability of the control and mitigation training procedures. The first author calculated the average acceptability rating for the control and mitigation conditions separately by summing ratings across each condition and dividing that sum by the total number of completed questionnaires. Caregiver acceptability of the training procedures was slightly higher for the mitigation condition (4.6 out of 5) relative to the control condition (4.4 out of 5).

\section{CHAPTER 2: RESULTS}

The first author reviewed caregiver-integrity data from the first five sessions of the renewal test when the caregiver or therapist fed the child using the function-based extinction protocol to ensure that changes in mean inappropriate mealtime behavior per minute were not a function of low feeder integrity. During the renewal test for the control condition, mean correct utensil placement was $99 \%$ (range, $98 \%$ to $100 \%$ ), mean correct procedure was $99 \%$ (range, $80 \%$ to $100 \%$ ), and mean incorrect attention was $0 \%$ across feeders. During the renewal test for the 
mitigation condition, mean correct utensil placement was 99\% (range, $99 \%$ to $100 \%$ ), mean correct procedure was $98 \%$ (range, $80 \%$ to $100 \%$ ), and mean incorrect attention was $0.6 \%$ (range, $0 \%$ to $20 \%$ ) across feeders.

Figure 1 shows mean inappropriate mealtime behavior per minute during the control (top) and mitigation (bottom) conditions for John (top left), Maisy (top right), Hope (bottom left), and Emilia (bottom right). For John during function-based reinforcement in Context A, rate of inappropriate mealtime behavior was high and variable in the control condition with solids $(M=$ 67; range, 43 to 86 ) and was initially low and stable but then increased and was high and variable in the mitigation condition with liquids ( $M=65$; range, 23 to 132). During function-based extinction in Context B, rate of inappropriate mealtime behavior decreased to zero in the control condition with solids $(M=20$; range, 0 to 55$)$ and decreased and was low and stable in the mitigation condition with liquids ( $M=3$; range, 0 to 27$)$. During the renewal test, rate of inappropriate mealtime behavior increased and was initially low and variable before decreasing to zero in the control condition with solids $(M=9$; range, 0 to 21$)$ and increased slightly in the first session before decreasing to zero in the mitigation condition with liquids ( $M=0.4$; range, 0 to 4$)$.

For Maisy during function-based reinforcement in Context A, rate of inappropriate mealtime behavior was moderately high and variable in the control condition with liquids $(M=$ 26; range, 24 to 29) and in the mitigation condition with solids ( $M=25$; range, 7 to 45$)$. During function-based extinction in Context $\mathrm{B}$, rate of inappropriate mealtime behavior was low and relatively stable in the control condition with liquids $(M=0.5$; range, 0 to 5$)$ and in the mitigation condition with solids ( $M=0.4$; range, 0 to 6$)$. During the renewal test, rate of inappropriate mealtime behavior increased initially and was low and variable before decreasing to zero in the control condition with liquids $(M=3$; range, 0 to 9$)$ and remained low and stable in the mitigation condition with solids ( $M=1$; range, 0 to 4$)$. 
For Hope during function-based reinforcement in Context A, rate of inappropriate mealtime behavior was moderately high and variable in the control condition with solids $(M=25$; range, 13 to 40) and high and increasing during the mitigation condition with liquids $(M=37$; range, 21 to 52). During function-based extinction in Context B, rate of inappropriate mealtime behavior decreased and was low and stable in the control condition with solids $(M=0.3$; range, 0 to 6$)$ and in the mitigation condition with liquids $(M=0.3$; range, 0 to 4$)$. During the renewal test, rate of inappropriate mealtime behavior remained at zero in the control condition with solids and in the mitigation condition with liquids.

For Emilia during function-based reinforcement in Context A, rate of inappropriate mealtime behavior was moderately high and variable in the control condition with Set 1 foods $(M$ $=42$; range, 22 to 77$)$ and in the mitigation condition with Set 2 foods $(M=34$; range, 9 to 61$)$. During function-based extinction in Context B, rate of inappropriate mealtime behavior decreased and was low and relatively stable in the control condition with Set 1 foods $(M=0.7$; range, 0 to 7) and mitigation condition with Set 2 foods ( $M=0.7$; range, 0 to 6$)$. During the renewal test, rate of inappropriate mealtime behavior remained at zero in the control condition with Set 1 foods and increased slightly in the first session then decreased to zero in the mitigation condition with Set 2 foods ( $M=0.7$; range, 0 to 4$)$. 

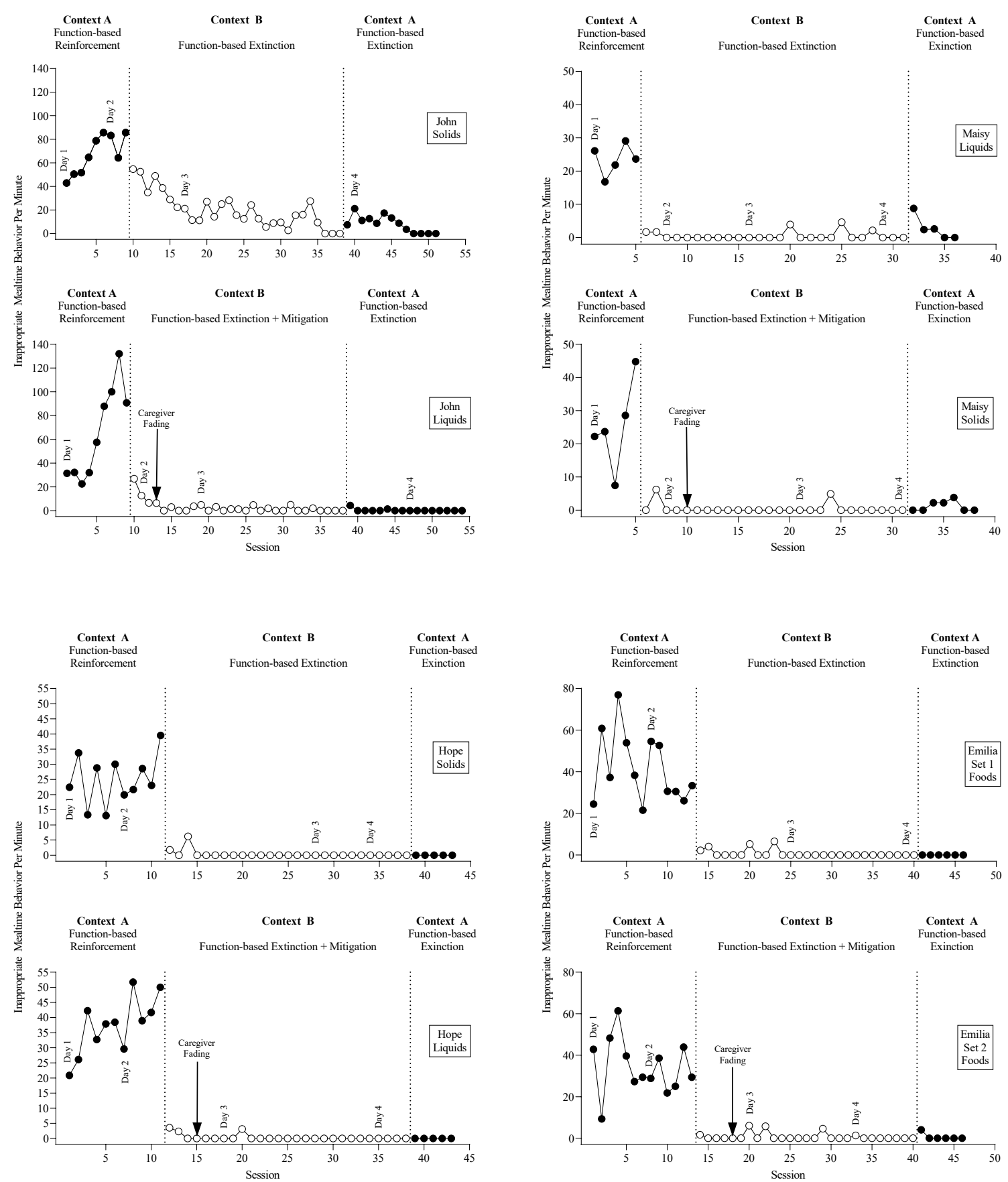

Figure 1. Rate of Inappropriate Mealtime Behavior for John, Maisy, Hope, and Emilia. Inappropriate mealtime behavior per minute during the control (top) and mitigation (bottom) conditions for John (top left), Maisy (top right), Hope (bottom left), and Emilia (bottom right). 
Figure 2 shows mean inappropriate mealtime behavior per minute during the control (top) and mitigation conditions (bottom), respectively, for Diego. During function-based reinforcement in Context A, rate of inappropriate mealtime behavior was high and variable in the control condition with Set 1 foods $(M=77$; range, 32 to 103$)$ and in the mitigation condition with Set 3 foods $(M=90$; range, 62 to 115$)$. During function-based extinction in Context $\mathrm{B}$, rate of inappropriate mealtime behavior decreased and was moderately high and variable initially then decreased and was low and stable in the control condition with Set 2 foods $(M=4$; range, 0 to 52$)$ and decreased and was moderately high and variable then decreased to zero in the mitigation condition with Set 4 foods $(M=10$; range, 0 to 60$)$. During the renewal test, rate of inappropriate mealtime behavior remained low and stable in the control condition with Set 1 foods $(M=0)$ and in the mitigation condition with Set 3 foods $(M=1$; range, 0 to 7$)$. 


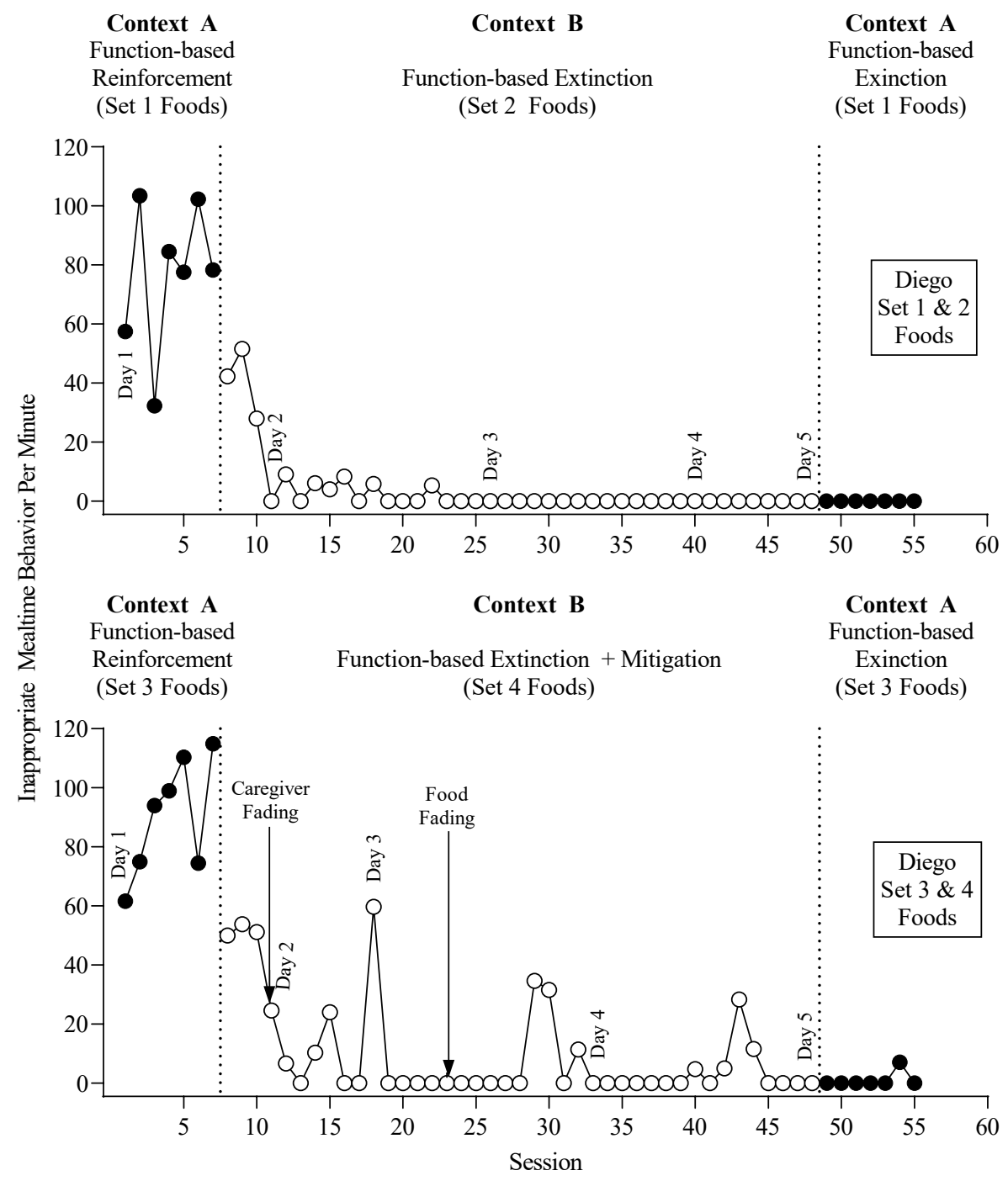

Figure 2. Rate of Inappropriate Mealtime Behavior for Diego.

Inappropriate mealtime behavior per minute during the control (top) and mitigation (bottom) conditions for Diego. 
Figure 3 shows mean inappropriate mealtime behavior per minute the control (top) and mitigation (bottom) conditions, respectively, for Jade. During function-based reinforcement in Context A, rate of inappropriate mealtime behavior was variable in the control condition with liquids ( $M=26$; range, 5 to 66$)$ and in the mitigation condition with solids ( $M=18$; range, 5 to 58). During function-based extinction in Context B, rate of inappropriate mealtime behavior was low and variable then decreased and was low and stable in the control condition with liquids $(M=$ 2; range, 0 to 20) and decreased and was low and variable then stabilized at zero in the mitigation condition with solids ( $M=1$; range 0 to 11$)$. During the renewal test, rate of inappropriate mealtime behavior increased and was low and variable in the control condition with liquids $(M=$ 3; range, 0 to 6 ) and remained at zero in the mitigation condition with solids. 


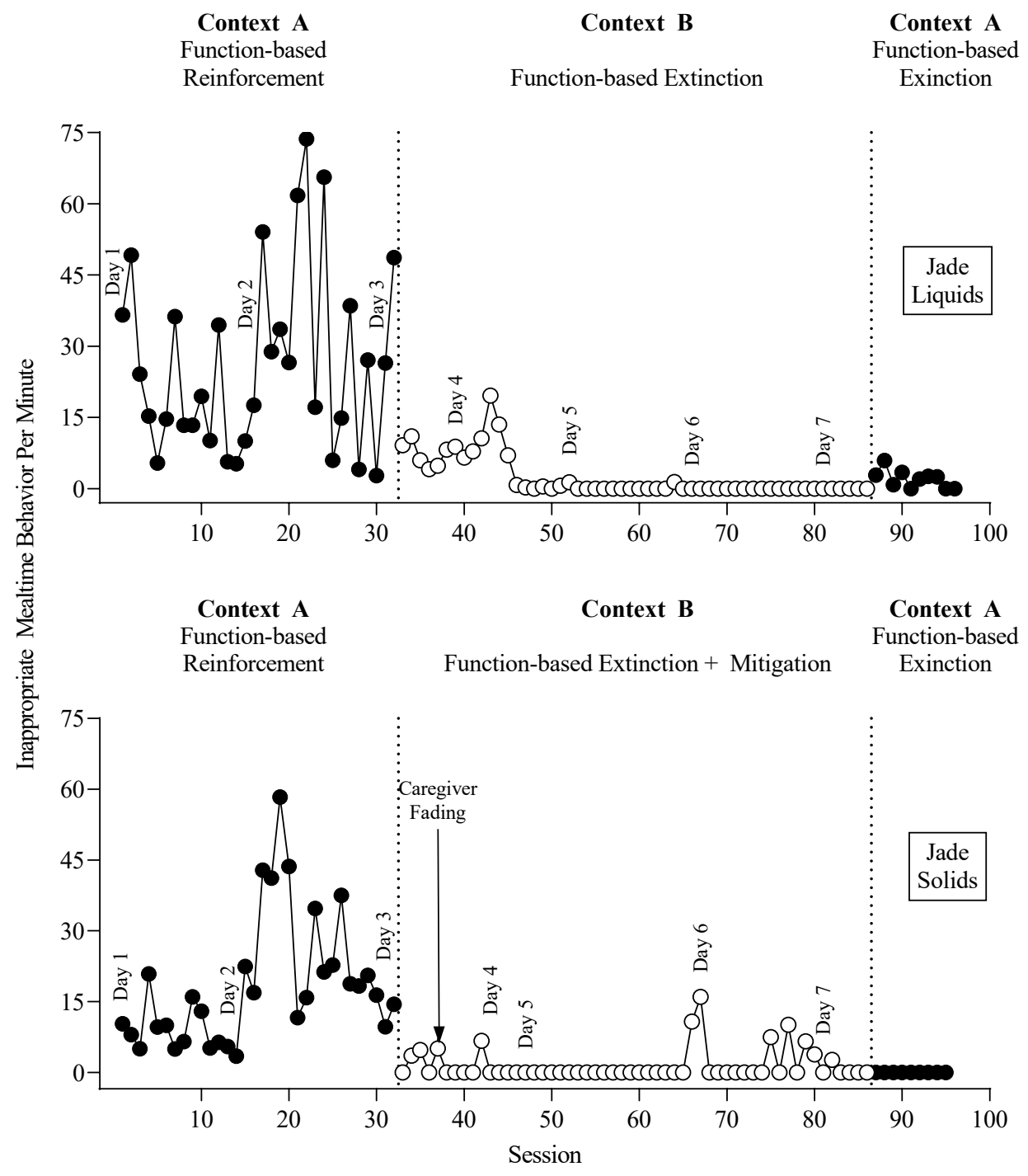

Figure 3. Rate of Inappropriate Mealtime Behavior for Jade. Inappropriate mealtime behavior per minute during the control (top) and mitigation (bottom) conditions for Jade. 
Figure 4 shows mean inappropriate mealtime behavior per minute during the control (top) and mitigation (bottom) conditions for Julian. During function-based reinforcement in Context A, rate of inappropriate mealtime behavior was high and variable in the control condition with Set 1 foods $(M=52$; range, 33 to 78$)$ and in the mitigation condition with Set 2 foods $(M=60$; range, 43 to 86). During function-based extinction in Context B, rate of inappropriate mealtime behavior decreased and was low and variable in the control condition with Set 1 foods $(M=5$; range, 0 to 26) and mitigation condition with Set 2 foods $(M=4$; range, 0 to 14). During the renewal test, rate of inappropriate mealtime behavior remained low and variable in the control condition with Set 1 foods $(M=7$; range, 0 to 13$)$ and remained at zero in the mitigation condition with Set 2 foods. 


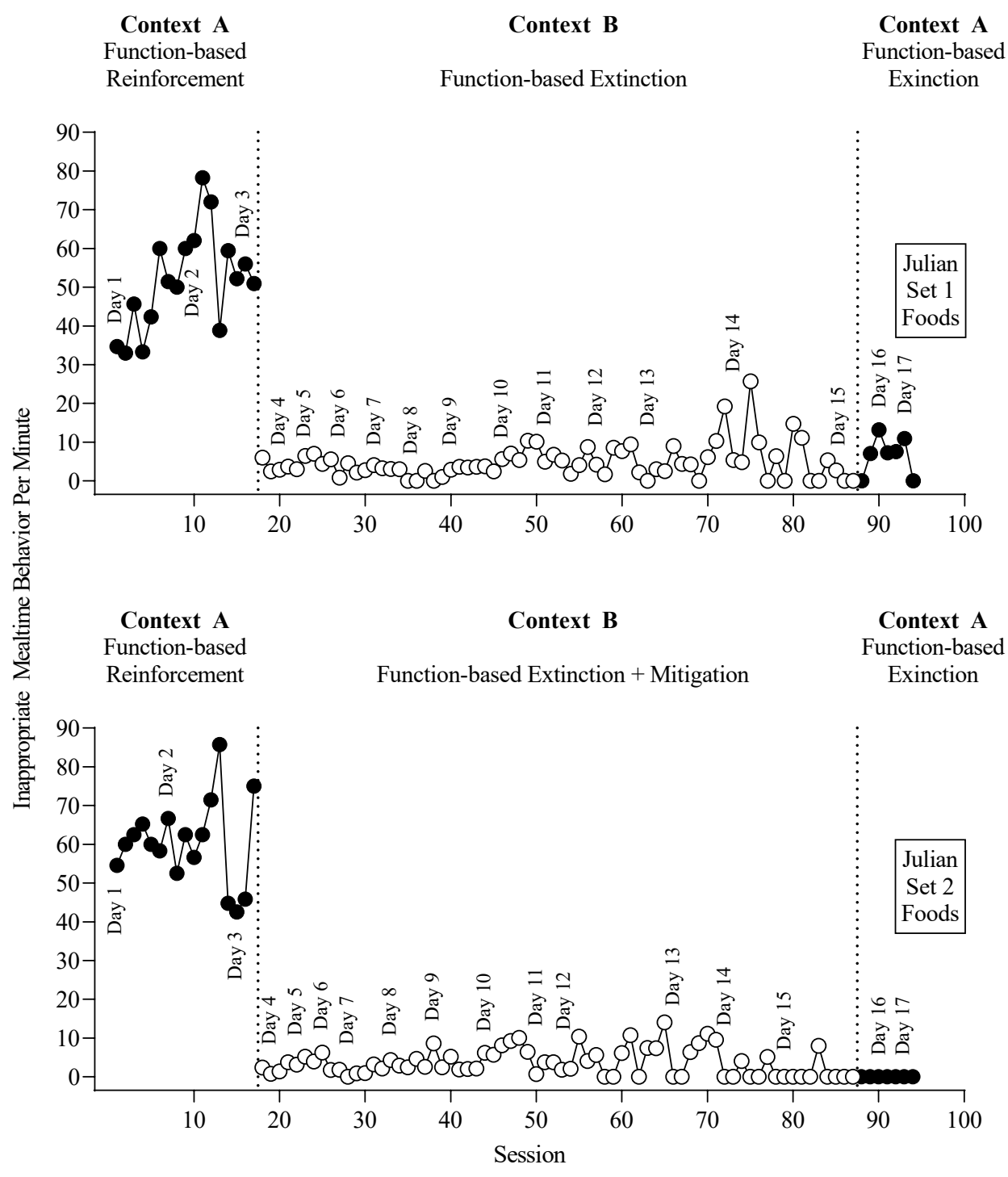

Figure 4. Rate of Inappropriate Mealtime Behavior for Julian. Inappropriate mealtime behavior per minute during the control (top) and mitigation (bottom) conditions for Julian. 
Figure 5 shows mean percentage of acceptance during the control (top) and mitigation (bottom) conditions for John (top left), Maisy (top right), Hope (bottom left), and Emilia (bottom right). For John during function-based reinforcement in Context A, mean percentage of acceptance was zero in the control condition with solids and in the mitigation condition with liquids. During function-based extinction in Context B, mean percentage of acceptance remained low and stable initially then increased to $100 \%$ during the control condition with solids $(M=$ $51 \%$; range, $0 \%$ to $100 \%$ ) and increased to moderately high and variable levels then stabilized at $80 \%$ during the mitigation condition with liquids $(M=59 \%$; range, $0 \%$ to $100 \%)$. During the renewal test, mean percentage of acceptance remained high and relatively stable in the control condition with solids $(M=93 \%$; range, $60 \%$ to $100 \%)$ and high and variable in the mitigation condition with liquids $(M=84 \%$; range, $40 \%$ to $100 \%)$.

For Maisy during function-based reinforcement in Context A, mean percentage of acceptance was zero in the control condition with liquids and variable in the mitigation condition with solids ( $M=40 \%$; range, $0 \%$ to $100 \%)$. During function-based extinction in Context B, mean percentage of acceptance was high and relatively stable in the control condition with liquids $(M=$ $95 \%$; range, $60 \%$ to $100 \%$ ) and in the mitigation condition with solids ( $M=98 \%$; range, $80 \%$ to $100 \%)$. During the renewal test, mean percentage of acceptance remained high and relatively stable in the control condition with liquids ( $M=96 \%$; range, $80 \%$ to $100 \%)$ and in the mitigation condition with solids $(M=97 \%$; range, $80 \%$ to $100 \%)$.

For Hope during function-based reinforcement in Context A, mean percentage of acceptance was low and stable in the control condition with solids ( $M=2 \%$; range, $0 \%$ to $20 \%)$ and the mitigation condition with liquids $(M=2 \%$; range, $0 \%$ to $20 \%$ ). During function-based extinction in Context B, mean percentage of acceptance increased and was high and stable in the control condition with solids $(M=97 \%$; range, $40 \%$ to $100 \%)$ and the mitigation condition with 
liquids $(M=95 \%$; range, $50 \%$ to $100 \%)$. During the renewal test, mean percentage of acceptance remained at $100 \%$ in the control and mitigation conditions.

For Emilia during function-based reinforcement in Context A, mean percentage of acceptance was zero in the control condition with Set 1 foods and mitigation condition with Set 2 foods. During function-based extinction in Context B, mean percentage of acceptance was low initially then increased and was high and stable in the control condition with Set 1 foods $(M=$ $93 \%$; range, $0 \%$ to $100 \%$ ) and increased and was high and relatively stable in the mitigation condition with Set 2 foods $(M=95 \%$; range, $0 \%$ to $100 \%)$. During the renewal test, mean percentage of acceptance remained at $100 \%$ in the control and mitigation conditions. 

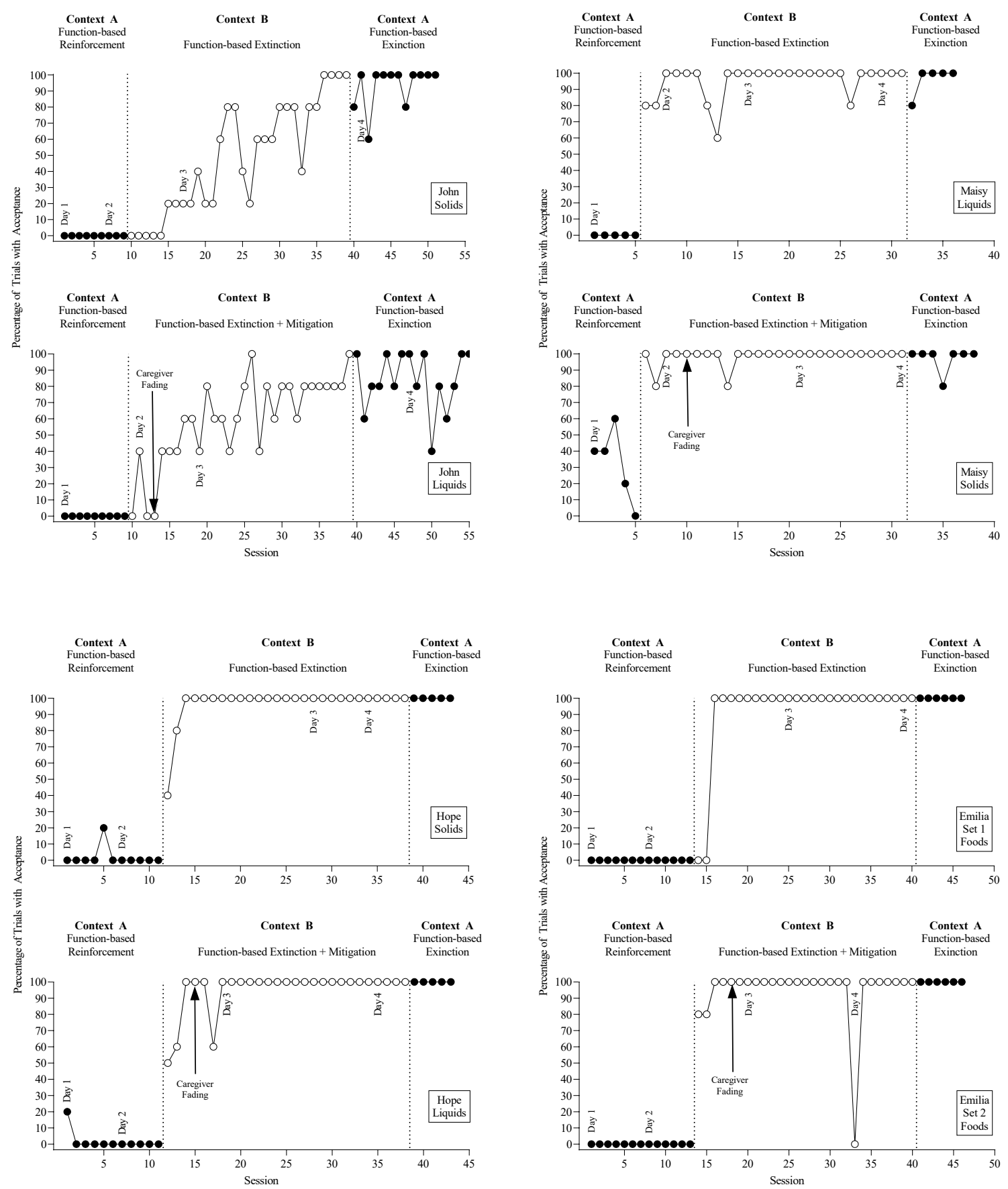

Figure 5. Percentage of Acceptance for John, Maisy, Hope, and Emilia. Percentage of trials with acceptance during the control (top) and mitigation (bottom) conditions for John (top left), Maisy (top right), Hope (bottom left), and Emilia (bottom right). 
Figure 6 shows mean percentage of acceptance during the control (top) and mitigation (bottom) conditions for Diego. During function-based reinforcement in Context A, mean percentage of acceptance was low and initially variable then decreased to zero during the control condition with Set 1 foods $(M=6 \%$; range, $0 \%$ to $20 \%)$ and remained at zero in the mitigation condition with Set 3 foods. During function-based extinction in Context B, mean percentage of acceptance remained low initially then increased to high stable levels in the control condition with Set 2 foods ( $M=92 \%$; range, $0 \%$ to $100 \%$ ) and remained low initially then increased to high relatively stable levels in the mitigation condition with Set 4 foods $(M=91 \%$; range, $0 \%$ to $100 \%$ ). During the renewal test, mean percentage of acceptance remained at $100 \%$ in the control and mitigation conditions. 


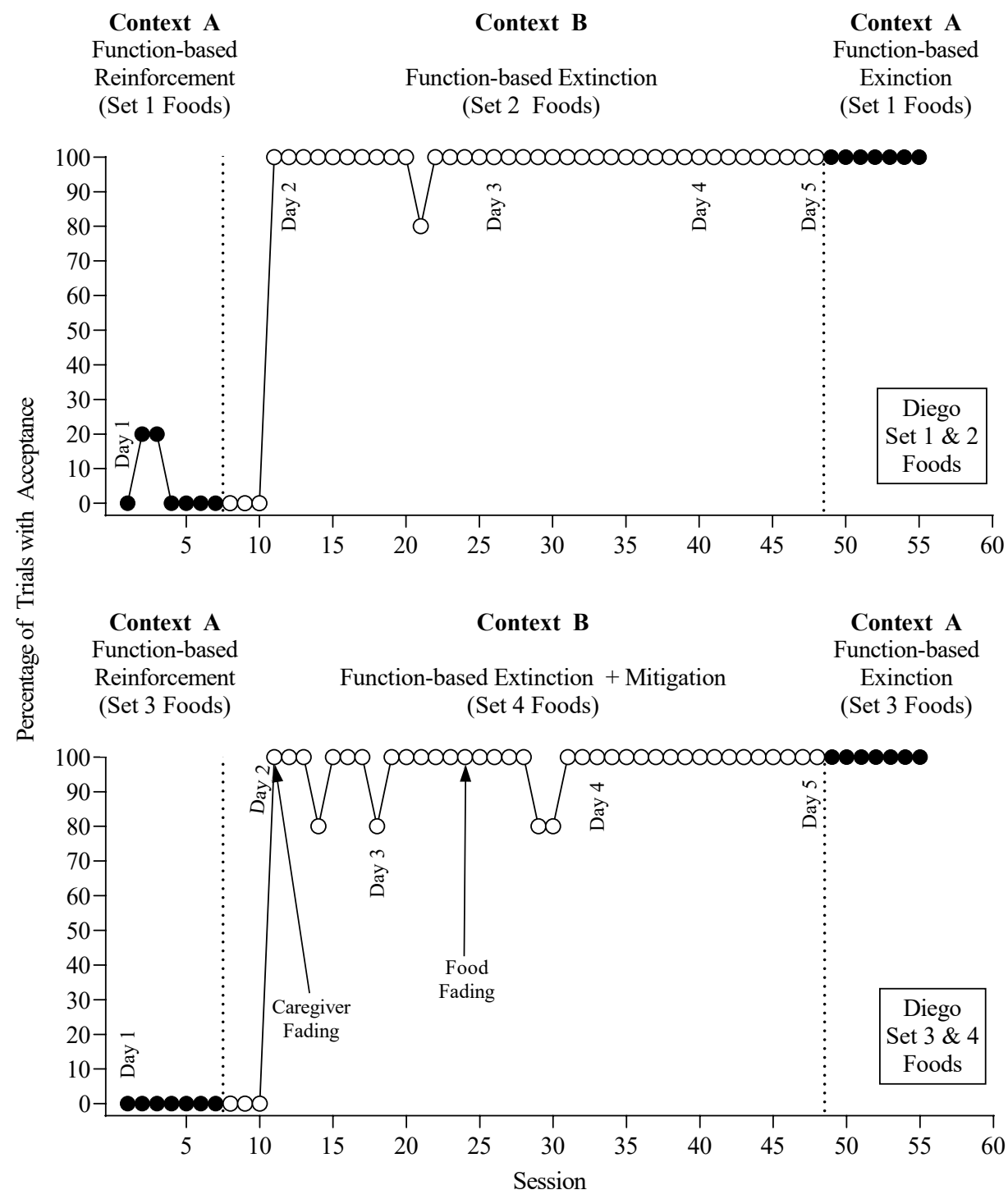

Figure 6. Percentage of Acceptance for Diego. Percentage of trials with acceptance during the control (top) and mitigation (bottom) conditions for Diego. 
Figure 7 shows mean percentage of acceptance during the control (top) and mitigation (bottom) conditions for Jade. During function-based reinforcement in Context A, mean percentage of acceptance was zero in the control condition with liquids and moderately low and variable in the mitigation condition with solids $(M=20 \%$; range, $0 \%$ to $80 \%)$. During functionbased extinction in Context B, mean percentage of acceptance was low and stable then increased and was high and variable in the control condition with liquids $(M=51 \%$; range, $0 \%$ to $100 \%)$ and was high and variable in the mitigation condition with solids $(M=96 \%$; range, $60 \%$ to 100$)$. During the renewal test, mean percentage of acceptance was moderately high and variable in the control condition with liquids $(M=58 \%$; range, $0 \%$ to $100 \%)$ and high and relatively stable in the mitigation condition with solids $(M=96 \%$; range, $80 \%$ to $100 \%)$. 


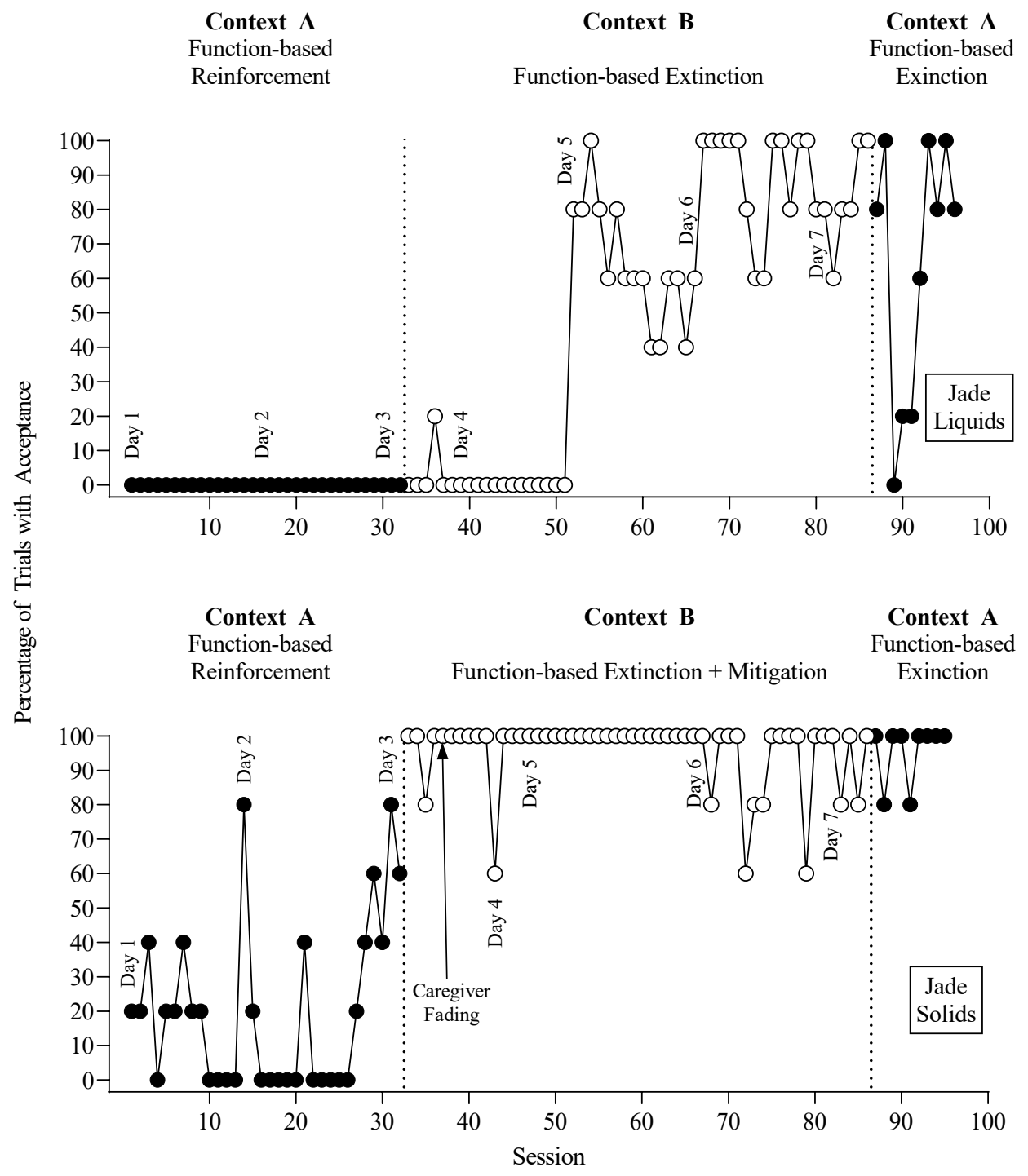

Figure 7. Percentage of Acceptance for Jade. Percentage of trials with acceptance during the control (top) and mitigation (bottom) conditions for Jade. 
Figure 8 shows mean percentage of acceptance during the control (top) and mitigation (bottom) conditions for Julian. During function-based reinforcement in Context A, mean percentage of acceptance was zero in the control condition with Set 1 foods and the mitigation condition with Set 2 foods. During function-based extinction in Context B, mean percentage of acceptance was low initially then increased and was high and variable in the control condition with Set 1 foods $(M=46 \%$; range, $0 \%$ to $100 \%)$ and increased and was high and stable in the mitigation condition with Set 2 foods $(M=49 \%$; range, $0 \%$ to $100 \%)$. During the renewal test, mean percentage of acceptance was high and stable in the control condition with Set 1 foods $(M=$ $97 \%$; range, $80 \%$ to $100 \%$ ) and remained at $100 \%$ in the mitigation condition with Set 2 foods. 
Context A

Function-based

Reinforcement
Context B

Function-based Extinction
Context A

Function-based Exinction

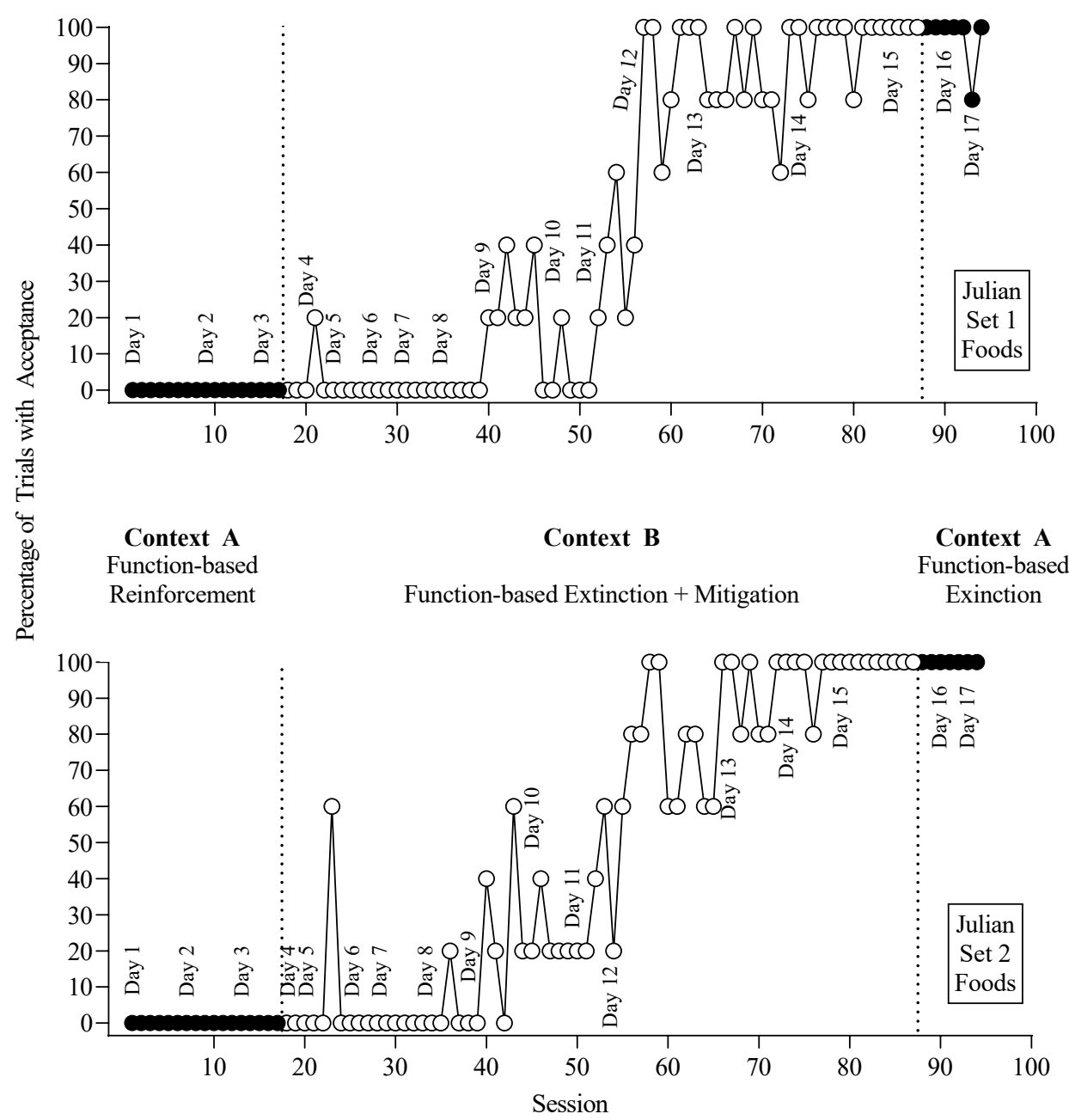

Figure 8. Percentage of Acceptance for Julian. Percentage of trials with acceptance during the control (top) and mitigation (bottom) conditions for Julian. 
Figure 9 shows proportion of baseline rate of inappropriate mealtime behavior during the renewal test in the control and mitigation conditions for each participant. We calculated proportion of baseline rate of inappropriate mealtime behavior in the control and mitigation conditions to compare our results with those of other studies. The first author calculated proportion of baseline rate during the renewal test by dividing the first five sessions of the renewal test by the mean rate of inappropriate mealtime behavior for the entire phase of functionbased reinforcement in Context A. We used the first five sessions of the renewal test because five was the fewest number of sessions we conducted for the renewal test across the control and mitigation conditions for all participants. The proportion of baseline rate was between 0 and 0.4 during the control condition and between 0 and 0.2 during the mitigation condition across participants. 


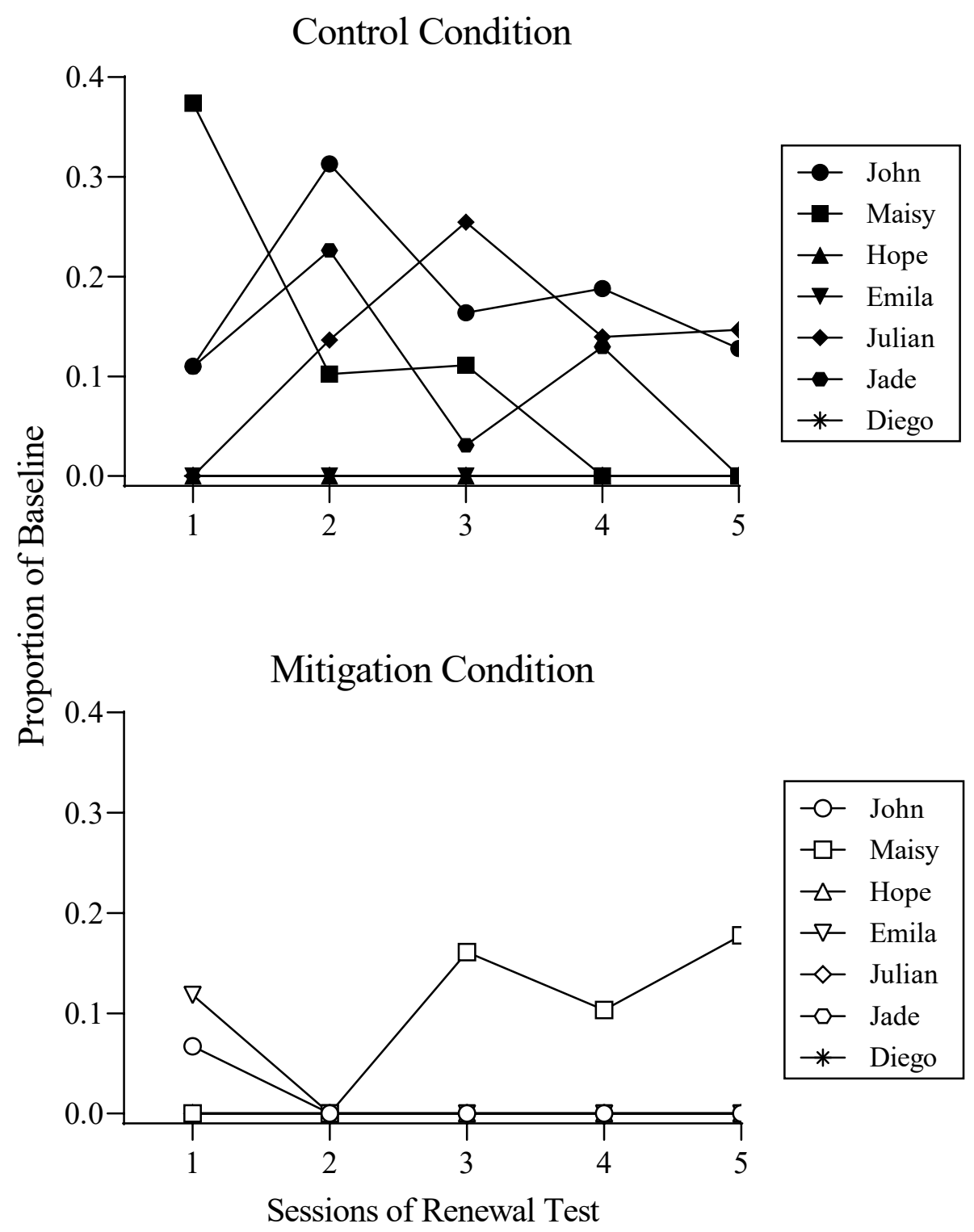

Figure 9. Proportion of Baseline. Proportion of baseline rate of inappropriate mealtime behavior during the renewal test in the control (top) and mitigation (bottom) conditions across participants. 


\section{CHAPTER 3: DISCUSSION}

Renewal effects are problematic because context changes, such as the caregiver implementing the intervention in the home, are often necessary for the generality and long-term maintenance of intervention effects. In the current study, we evaluated renewal of negatively reinforced socially significant behavior following clinically relevant context changes during extinction using an $\mathrm{ABA}$ renewal arrangement. Results of translational investigations on $\mathrm{ABA}$ renewal of socially significant behavior have demonstrated renewal of positively and negatively reinforced behavior such as severe problem behavior (Kelley et al., 2018; Pritchard et al., 2016; Saini et al., 2018), undesirable caregiver behavior (Mitteer et al., 2018), and inappropriate mealtime behavior (Ibañez et al., 2019; Kelley et al., 2018) following changes in implementer, setting, and implementer and setting combined. We extended the findings of Ibañez et al. (2019) and Kelley et al. (2018) by evaluating renewal of inappropriate mealtime behavior following changes in the feeder from therapist to caregiver, setting from clinic to home, food, and a combination of these changes.

Researchers have shown that multiple context training (Bandarian-Balooch \& Neumann, 2011; Bernal-Gamboa, Nieto, et al., 2017; Kelley et al., 2018; Nieto et al., 2017), context similarity (Bandarian-Balooch \& Neumann, 2011; Todd et al., 2012), and introducing discriminative stimuli during intervention and presenting these stimuli in other training contexts (Bernal-Gamboa, Gámez, et al., 2017; Kelley et al., 2018; Nieto et al., 2017; Todd et al., 2012) may effectively prevent renewal of operant behavior. However, research evaluating the efficacy of these procedures with socially significant behavior, like inappropriate mealtime behavior, is limited. Only one study to our knowledge has evaluated renewal-mitigation procedures for renewal of socially significant behavior. Kelley et al. (2018) found that multiple context training was effective at preventing renewal of aggression following a change in setting and implementer. Kelley et al. also found that therapist-caregiver pairing mitigated renewal of inappropriate 
mealtime behavior for one participant and decreased the magnitude and persistence of renewal for the other participant following a change in implementer from therapist to caregiver during extinction. We extended Kelley et al. in three ways. First, we evaluated renewal and renewalmitigation of inappropriate mealtime behavior concurrently instead of sequentially. Second, we evaluated systematic fading as a renewal-mitigation procedure for inappropriate mealtime behavior following a change in the feeder from therapist to caregiver. We gradually increased the caregiver's involvement in implementing components of the extinction procedure (e.g., keeping the spoon at the lips) to provide more opportunities for the caregiver to develop stimulus control for extinction of inappropriate mealtime behavior. Third, we evaluated the efficacy of context similarity and the combination of systematic fading and context similarity as a renewal-mitigation procedure for inappropriate mealtime behavior following changes in setting, feeder and setting, and feeder and food. This is the first study to our knowledge to evaluate systematic fading and context similarity alone and in combination as renewal-mitigation procedures for socially significant behavior.

We assessed renewal and renewal-mitigation by analyzing inappropriate mealtime behavior in terms of rate, immediacy, and persistence during the renewal test in the control and mitigation conditions. We evaluated renewal of inappropriate mealtime behavior following a change in the feeder from therapist to caregiver during intervention for solids and liquids refusal for John, Maisy, Hope; and solids refusal for Emilia. Researchers have shown that caregivers of children with feeding difficulties provide escape from bites and drinks in the form of breaks or meal termination, attention, or access to tangible items contingent on inappropriate mealtime behavior (Borrero et al., 2010; Piazza, Fisher, et al., 2003), and these consequences may function as reinforcers for inappropriate mealtime behavior (Bachmeyer et al., 2019; Najdowski et al., 2008; Piazza et al., 2003). Over time, the caregiver may signal reinforcement availability for inappropriate mealtime behavior and this stimulus control could compete with the extinction 
contingency when the caregiver implements extinction of inappropriate mealtime behavior. The caregiver is often a necessary context change during intervention for inappropriate mealtime behavior; therefore, it is particularly relevant to evaluate renewal-mitigation procedures to prevent renewal when the caregiver implements the intervention with the child.

We used systematic fading during function-based extinction in Context B of the mitigation condition by gradually fading the caregiver in as the feeder for John, Maisy, Hope, and Emilia. Researchers have shown that renewal may be less likely when stimuli that signal extinction of the target behavior, such as a colored card, are present in contexts previously associated with reinforcement of the target behavior (Kelley et al., 2018; Nieto et al., 2017; Podlesnik et al., 2017; Todd et al., 2012), perhaps because these stimuli signal extinction of the target behavior in other contexts or they develop inhibitory control over the target behavior (Podlesnik et al., 2017; Todd, 2013; Todd et al., 2014).

For John, we observed renewal of inappropriate mealtime behavior during the renewal test in the control condition with solids and the mitigation condition with liquids. We observed an immediate renewal effect during the control and mitigation conditions. However, rate of inappropriate mealtime behavior was higher and persisted across more renewal-test sessions in the control condition (nine sessions) relative to the mitigation condition (one session). We also observed a decrement in percentage of acceptance during the renewal test of the control and mitigation conditions, with more variability and more sessions below clinically acceptable levels (i.e., $80 \%$ ) during the mitigation condition relative to the control condition. John had a history of drinking formula from a bottle or sippy cup only and consuming caregiver-fed bites of pureed foods from a spoon (i.e., yogurt, applesauce) for three years before admission to our program. Consequently, John may have had a relatively long reinforcement history for refusal of pureed foods from a spoon and short reinforcement history for refusal of liquids from an open cup, which may have contributed to his results. 
For Maisy, we observed renewal of inappropriate mealtime behavior during the renewal test in the control condition with liquids and mitigation condition with solids. We observed an immediate renewal effect during the renewal test of the control condition and a delayed renewal effect during renewal test in the mitigation condition (i.e., third session). Additionally, rate of inappropriate mealtime behavior during the renewal test was generally higher in the control condition relative to the mitigation condition. For Maisy, we observed a decrement in percentage of acceptance during the first renewal-test session in the control condition and the fourth renewaltest session in the mitigation condition, although these decrements were still at clinically acceptable levels (i.e., 80\%). Procedural integrity for Maisy's mother decreased to $80 \%$ during the third and fourth renewal-test sessions in the mitigation condition, which may explain the increase in inappropriate mealtime behavior during these sessions (St. Peter Pipkin et al., 2010). The data-collection procedure did not provide information about whether these integrity errors were errors of commission or omission.

For Hope, we did not observe renewal of inappropriate mealtime behavior during the renewal test in the control condition with solids or mitigation condition with liquids. We also did not observe a decrement in percentage of acceptance during the renewal test in the control and mitigation conditions. For Emilia, we did not observe renewal during the renewal test in the control condition with Set 1 foods but did observe renewal during the renewal test in the mitigation condition with Set 2 foods. However, rate of inappropriate mealtime behavior was low and did not persist longer than one session during the renewal test of the mitigation condition. We did not observe a decrement in percentage of acceptance during the renewal test in the control and mitigation conditions for Emilia.

We analyzed the efficacy of systematic caregiver fading with participants for which we observed renewal during the renewal test of the control condition (i.e., John and Maisy). During the renewal test, rate of inappropriate mealtime behavior was lower for John and Maisy and 
persisted across fewer sessions for John in the mitigation condition relative to the control condition. Our results suggest that systematic fading decreased the magnitude and persistence of renewal, but may not have prevented renewal for John and Maisy. Kelley et al. (2018) obtained similar results with one participant following their therapist-caregiver pairing procedure for renewal of inappropriate mealtime behavior.

For Diego, we evaluated renewal of inappropriate mealtime behavior following a change in feeder and food from therapist feeding Set 2 (control condition) and Set 4 (mitigation condition) foods to the caregiver feeding Set 1 (control condition) and Set 3 (mitigation condition) foods during intervention for solids refusal. These are common context changes we present during treatment for avoidant/restrictive food intake disorder and that caregivers often report as challenging. We used systematic fading to introduce the caregiver as the feeder and introduce food during function-based extinction in Context B.

We did not observe renewal of inappropriate mealtime behavior during the renewal test in the control and mitigation conditions with Set 1 and Set 3 foods, respectively. We also did not observe a decrement in percentage of acceptance during the renewal test of the control and mitigation conditions for Diego. One explanation for these findings is that the control and mitigation conditions were not discriminable to Diego because the feeder presented solids in both conditions. Although we used discriminative stimuli such as colored cards and bowls, pureed foods may be difficult to discriminate because they do not have as many distinctive properties as the same foods at table texture. For example, potato and cauliflower have similar visual properties at a pureed texture relative to these foods at table texture. Alternatively, there may have been carry-over effects from the caregiver and food fading procedures.

For Jade, we evaluated renewal of inappropriate mealtime behavior following changes in the feeder and setting from therapist in the clinic to caregiver in the home during intervention for solids and liquids refusal. We evaluated these context changes for Jade for several reasons. First, 
we observed low rates of inappropriate mealtime behavior and variable levels of acceptance during the therapist- and caregiver-conducted functional analysis for solids in the clinic. When Jade's mother conducted the functional analysis for solids in the home, we observed inappropriate mealtime behavior and decrements in acceptance; therefore, it was particularly relevant to evaluate a feeder and setting change for Jade because the caregiver feeding in the home seemed to be the necessary stimulus conditions under which inappropriate mealtime behavior occurred during bite presentations. Additionally, the combination of a feeder and setting change is common during treatment for avoidant/restrictive intake disorder because children feed in different environments (e.g., home, school) with many individuals (e.g., grandparents, peers).

We used systematic fading and context similarity together as a renewal-mitigation procedure during function-based extinction in Context B of the mitigation condition for Jade. Results of previous research suggest that increasing the similarity between acquisition and extinction contexts may mitigate renewal upon returning to the context associated with reinforcement of the target behavior (Bandarian-Balooch \& Neumann, 2011; Todd et al., 2012). Additionally, renewal-mitigation procedures may be more effective when combined (BandarianBalooch \& Neumann, 2011; Bernal-Gamboa, Nieto, et al., 2017; Krisch et al., 2018); however this research is limited, particularly with renewal of socially significant behavior.

For Jade, We observed renewal of inappropriate mealtime behavior in the control condition with liquids only, which replicated the findings of Ibañez et al. (2019) and Saini et al. (2018) who observed renewal of socially significant behavior (inappropriate mealtime behavior and destructive behavior, respectively) following a change in implementer and setting during extinction. Jade's results, along with the findings of Ibañez et al. and Saini et al., demonstrate that changes in the implementer and setting produce renewal of negatively reinforced, socially significant behavior. We did not observe renewal during the renewal test of the mitigation condition with solids, indicating that the combination of systematic fading and context similarity 
during extinction in Context B prevented renewal of inappropriate mealtime behavior following changes in the feeder and setting.

We observed a decrement in percentage of acceptance during the first and second renewal-test sessions of the control and mitigation conditions respectively. However, percentage of acceptance decreased below clinically acceptable levels (i.e., below $80 \%$ ) during the renewal test in the control condition only. One explanation for Jade's results is that liquids may have been more difficult or aversive than solids because inappropriate mealtime extinguished and acceptance increased more slowly during function-based extinction in Context B with liquids relative to solids. Jade also accepted bites during the functional analysis and during functionbased reinforcement in her home, which may suggest that solids were more preferred than liquids or that liquids were more aversive or difficult for Jade.

For Julian, we evaluated renewal of inappropriate mealtime behavior following a setting change from clinic to a simulated home during intervention for solids refusal. We evaluated a simulated home setting as the context change for Julian because his caregivers could not attend sessions and we wanted to evaluate renewal during another common context change children experience during treatment and that caregivers report as challenging. We observed renewal of inappropriate mealtime behavior during the renewal test of the control condition with Set 1 foods only for Julian, which was similar to the renewal effects observed by Mitteer et al. (2018) with undesirable caregiver behavior following a setting change. Julian's results, along with the results of Mitteer et al., suggest that changes in the setting during extinction may evoke renewal of negatively reinforced socially significant behavior.

We evaluated context similarity during function-based extinction in Context B as a renewal-mitigation procedure for inappropriate mealtime behavior for Julian. We did not observe renewal of inappropriate mealtime behavior during the mitigation condition with Set 2 foods, indicating that context similarity prevented renewal of inappropriate mealtime behavior following 
changes in the setting. We observed a decrement in percentage of acceptance during the renewal test of the control condition only; however, this decrement occurred during the sixth renewal-test session and remained at clinically acceptable levels.

Interestingly, we observed the most robust renewal effects during the renewal test for Jade and Julian. The main commonality between Jade and Julian's renewal arrangement was a setting change; therefore, we wondered whether the setting exerts more control over inappropriate mealtime behavior than the caregiver. Results of our study and previous research show that a stimulus change such as a change in setting or implementer may exert more control over behavior than the contingency (e.g., Ibañez et al., 2019; Saini et al., 2018). Children in our study had a relatively short history of reinforcement for inappropriate mealtime behavior in the clinic and a more recent history of extinction for inappropriate mealtime behavior in the clinic. Therefore, the clinic may have signaled extinction of inappropriate mealtime behavior, which competed with the child's history of reinforcement with the caregiver. Caregivers of children with feeding disorders often report rigid mealtime routines, which may discourage caregivers from feeding their children in environments other than the home. Consequently, a majority of the child's reinforcement history for inappropriate mealtime behavior with the caregiver likely occurs in the home. Alternatively, there may be more stimuli in the home that signal reinforcement availability (e.g., mealtime chair, kitchen decorations) relative to the presence of the caregiver as a signal for reinforcement availability in the clinic. However, it is unclear which stimuli in a given context an individual attends to; therefore, it is difficult to determine the contextual stimuli that exert control over behavior following setting changes during extinction.

\section{General Discussion}

We observed renewal of inappropriate mealtime behavior following a change in feeder from therapist to caregiver for two of four participants, feeder and setting from therapist in clinic to caregiver in the home with one participant, and setting from clinic to simulated home for one 
participant despite high levels of integrity. Our results replicate the findings of previous research demonstrating that a change in implementer (Kelley et al., 2018), implementer and setting (Ibañez et al., 2019; Saini et al., 2018), and setting (Mitteer et al., 2018) can produce renewal of negatively reinforced socially significant behavior. These findings are important because these context changes are often necessary for treatment generality and maintenance and are changes that caregivers reports as most challenging, particularly during treatment for avoidant/restrictive food intake disorder.

In general, when we observed renewal during the renewal test in the control or mitigation conditions, we observed inappropriate mealtime behavior immediately following the context change. By contrast, inappropriate mealtime behavior returned during the second renewal-test session in the control condition for Julian and the third renewal-test session in the mitigation condition for Maisy; however, the increase in inappropriate mealtime behavior during the renewal test in the mitigation condition may have been due to an integrity error for Maisy. Other researchers have reported similar response patterns during renewal evaluations with humans; however, researchers differ in categorizing delayed responding as renewal (Saini \& Mitteer, 2019 for discussion). For example, Ibañez et al. (2019) and Kelley et al. (2018) observed inappropriate mealtime behavior during the fourth renewal-test session for one participant and interpreted this as renewal. By contrast, Liddon et al. (2018) observed reemergence of task completion during the second renewal-test session for one participant and did not interpret this as renewal. Researchers may also differ in categorizing modest increases in target behavior during the renewal test as renewal. For example, we observed a slight increase in inappropriate mealtime behavior during the first renewal-test session of the mitigation condition for John and interpreted this as renewal. By contrast, Kimball et al. (2020) and Saini et al. (2018) observed similarly modest increases in target responding during the first renewal-test session for one participant and did not interpret this as renewal. As Saini and Mitteer (2019) discussed, researchers have not agreed upon a definition 
for renewal; therefore, interpretations of renewal effects may differ across researchers. Differences in renewal definitions may pose challenges for comparing renewal effects across studies and interpreting the efficacy of renewal-mitigation procedures.

Results of previous research have suggested that behavior that is more resistant to extinction initially may be more likely to reemerge following a context change during extinction (Podlesnik \& DeLeon, 2015). Of the children for which we observed renewal in the control condition (i.e., John, Maisy, Jade, Julian), inappropriate mealtime behavior was more resistant to extinction initially during function-based extinction in Context B in the control condition relative to the mitigation condition for all participants. For example, the latency (in sessions) to the first session with zero inappropriate mealtime behavior was longer in the control condition relative to the mitigation condition during function-based extinction in Context B for John, Maisy, Jade, and Julian, although this difference was small for Maisy (i.e., two sessions). Researchers have also suggested that behavior that is more persistent during extinction may be more likely to relapse following context changes during extinction (Kimball et al., 2018; Podlesnik et al., 2017). In the current study, inappropriate mealtime behavior occurred during more sessions of extinction in Context B in the control condition relative to the mitigation condition for John, Maisy, Jade, and Julian, this difference was also small for Maisy (i.e., three sessions).

When rate of inappropriate mealtime behavior remained at zero during the renewal test of the control condition, acceptance remained high and stable, as evidenced by Hope, Emilia, and Diego. We observed this pattern during the renewal test in the mitigation condition with Hope, Diego, Jade, and Julian. Interestingly, when we observed renewal of inappropriate mealtime behavior during the renewal test of the control condition, we generally observed concomitant decrements in percentage of acceptance. This covariation effect is evidenced in the control condition data for John, Maisy, and Jade. Researchers have observed similar disruption of treatment effects between responses targeted for extinction and responses targeted for acquisition 
during ABA renewal arrangements with other socially significant behavior (Ibañez et al., 2019; Kelley et al., 2018; Saini et al., 2018). For example, Ibañez et al. (2019) observed renewal of inappropriate mealtime behavior and decrements in acceptance following a feeder and setting change during extinction. Kelley et al. (2018) observed renewal of negatively reinforced aggression and decrements in percentage of compliance following a change in therapist and setting despite continued availability of reinforcement for compliance during the renewal test. Similarly, Saini et al. (2018) observed renewal of negatively and positively reinforced destructive behavior and concomitant decrements in the functional communication response following a change in the implementer and setting despite the continued availability of reinforcement for the functional communication response. These results suggest that context changes such in the therapist, setting, or both during extinction may produce renewal of a previously extinguished target response and decrements in alternative appropriate responding, which is an important consideration for evaluating procedures to enhance the generality of treatment effects.

We evaluated proportion of baseline rate of inappropriate mealtime behavior during the renewal test in the control and mitigation conditions to quantify the amount of renewal and compare our results across participants and previous research. Proportion of baseline values closer to 1.0 demonstrate closer approximations to rate of inappropriate mealtime behavior during the entire phase of function-based reinforcement in Context A. We obtained greater values of proportion of baseline rate of inappropriate mealtime behavior during the renewal test of the control condition relative to the renewal test of the mitigation condition across participants except for Maisy. We compared our proportion of baseline data to that obtained by Ibañez et al. (2019) and Saini et al. (2018) who evaluated renewal of negatively reinforced socially significant behavior. We found that our proportion of baseline values were generally lower than those obtained by Ibañez et al. and Saini et al. 
We analyzed the efficacy of our renewal-mitigation procedures with participants for which we observed renewal of inappropriate mealtime behavior during the renewal test of the control condition. Systematic caregiver fading decreased the magnitude of renewal effects following a feeder change from therapist to caregiver but did not prevent renewal. The combination of systematic fading and context similarity mitigated renewal following a change in feeder and setting from therapist in the clinic to caregiver in the home and context similarity mitigated renewal following a change in setting from the clinic to a simulated home. Future research should further investigate the efficacy of these renewal-mitigation procedures with more children and the generality of them across other socially significant behavior.

One limitation of the study was the potential for carry-over effects from alternating between the control and mitigation conditions. We tried to increase the discriminability between the conditions by assigning solids or liquids to the control or mitigation condition for John, Maisy, Hope, and Jade and using colored stimuli (i.e., tablecloths, bowls, cards) for Emilia and Diego but the presence of the caregiver during some meals and not others may have decreased the likelihood or magnitude of renewal for some children. Similarly, we presented the same colored stimuli in each phase of the control and mitigation conditions for Emilia, Diego, and Julian. This history of contacting reinforcement and extinction in the presence of the colored stimuli may have decreased the discriminability between phases in each condition and the likelihood of renewal effects (Saini et al., 2018). An alternative explanation for our findings is that responding during the renewal test may have been influenced by order effects. We randomized the order of the renewal tests across participants but exposure to the renewal test in one condition may have influenced responding during the renewal test of the other condition.

Previous research has shown that longer acquisition periods (i.e., reinforcement) may increase the likelihood of renewal effects (Todd et al., 2012). Participants in the current study had a long history of contacting reinforcement for inappropriate mealtime behavior before admission 
to our day-treatment program ( 2 to 4.5 years), which may have contributed to renewal effects for some children. It is difficult to measure and control for a child's reinforcement history for inappropriate mealtime behavior before admission for treatment; therefore, relations between reinforcement history and renewal effects in the current study are speculative.

The results of the current study suggest that renewal may occur during intervention for inappropriate mealtime behavior for children with avoidant/restrictive food intake disorder, particularly following stimulus changes such as changes in the feeder from therapist to caregiver, setting from the clinic to the home, and a combination of these changes. This is concerning given that these are necessary context changes for the generality and long-term maintenance of intervention effects. Renewal-mitigation procedures such as systematic fading and context similarity alone and in-combination may be effective at preventing or decreasing the magnitude of renewal effects, but we need to further understand the conditions under which renewal of inappropriate mealtime behavior occurs during intervention and the conditions under which renewal-mitigation procedures will be efficacious. 


\section{BIBLIOGRAPHY}

American Psychiatric Association (2013). Diagnostic and statistical manual of mental disorders (5th ed.). Arlington, VA: American Psychiatric Publishing.

Bachmeyer, M. H., Kirkwood, C. A., Criscito, A. B., Mauzy, C. R., IV, \& Berth, D. P. (2019). A comparison of functional analysis methods of inappropriate mealtime behavior. Journal of Applied Behavior Analysis, 52(3), 603-621. https://doi.org/10.1002/jaba.556

Bachmeyer, M. H., Piazza, C. C., Fredrick, L. D., Reed, G. K., Rivas, K. D., \& Kadey, H. J. (2009). Functional analysis and treatment of multiply controlled inappropriate mealtime behavior. Journal of Applied Behavior Analysis, 42(1), 641-658.

https://doi.org/10.1901/jaba.2009.42641

Bandarian-Balooch, S. B., \& Neumann, D. L. (2011). Effects of multiple contexts and context similarity on the renewal of extinguished conditioned behavior in an ABA design with humans. Learning and Motivation, 42(1), 53-63.

https://doi.org/10.1016/j.brat.2012.06.003

Bernal-Gamboa, R., Gámez, A. M., \& Nieto, J. (2017). Reducing spontaneous recovery and reinstatement of operant performance through extinction-cues. Behavioural Processes, 135(2), 1-7. https://doi.org/10.1016/j.beproc.2016.11.010

Bernal-Gamboa, R., Nieto, J., \& Uengoer, M. (2017). Effects of extinction in multiple contexts on renewal of instrumental responses. Behavioural Processes, 142(9), 64-69. https://doi.org/10.1016/j.beproc.2017.06.003

Bryant-Waugh, R., Markham, L., Kreipe, R. E., \& Walsh, B. T. (2010). Feeding and Eating Disorders in Childhood. International Journal of Eating Disorders, 43(2), 98-111. https://doi.org/10.1002/eat.20795

Borrero, C. S. W., Woods, J. N., Borrero, J. C., Masler, E. A., \& Lesser, A. D. (2010). Descriptive analysis of pediatric food refusal and acceptance. Journal of 
Applied Behavior Analysis, 43(1), 71-88. https://doi.org/10.1901/jaba.2010.43-71

Bouton, M. E., Todd, T. P., Vurbic, D., \& Winterbaurer, N. E. (2011). Renewal after the extinction of free operant behavior. Learning \& Behavior, 39(1), 57-67. https://doi.org/10.3758/s13420-011-0018-6

Bullock, C. E., Fisher, W. W., \& Hagopian, L. P. (2017). Description and validation of a computerized behavioral data program: "BDataPro." The Behavior Analyst, 40(1), 275 285. https://doi.org/10.1007/s40614-016-0079-0

Centers for Disease Control and Prevention, National Center for Health Statistics. CDC growth charts: United States. http://www.cdc.gov/growthcharts/. May 30, 2000.

Cohenour, J. M., Volkert, V. M., \& Allen, K. D. (2018). An experimental demonstration of AAB renewal in children with autism spectrum disorder. Journal of Experimental Behavior Analysis, 110(1), 63-73. https://doi.org//10.1002/jeab.443

Freedman, D. S., Dietz, W. H., Srinivasan, S. R., \& Berenson, G. S. (1999). The relation of overweight to cardiovascular risk factors among children and adolescents: The Bogalusa Heart Study. Pediatrics, 103(6), 1175-1182. https://doi.org/10.1542/peds.103.6.1175

Girolami, P. A., \& Scotti, J. R. (2001). Use of analog functional analysis in assessing the function of mealtime behavior problems. Education and Training in Mental Retardation and Developmental Disabilities, 36(2), 207-223. https://www.jstor.org/stable/23879736

Ibañez, V., Piazza, C., \& Peterson, K. (2019). A translational evaluation of renewal of inappropriate mealtime behavior. Journal of Applied Behavior Analysis, 52(4), 10051020. https://doi.org/10.1002/jaba.647

Kelley, M. E., Jimenez-Gomez, C., Podlesnik, C. A., \& Morgan, A. (2018). Evaluation of renewal mitigation of negatively reinforced socially significant operant behavior. Learning and Motivation, 63(13), 133-441. https://doi.org/10.1016/j.lmot.2018.05.003 
Kelley, M. E., Liddon, C. J., Ribeiro, A., Greif, A. E., \& Podlesnik, C. A. (2015). Basic and translational evaluation of renewal of operant responding. Journal of Applied Behavior Analysis, 48(2), 390-401. https://doi.org/10.1002/jaba.209

Kimball, R. T., Greer, B. D., Randall, K. R., \& Briggs, A. M. (2020). Investigations of operant ABA renewal during differential reinforcement. Journal of the Experimental Analysis of Behavior, 113(1), 187-205. https://doi.org/10.1002/jeab.577

Kimball, R. T., Kelley, M. E., Podlesnik, C. A., Forton, A., \& Hinkle, B. (2018). Resurgence with and without and alternative response. Journal of Applied Behavior Analysis, 51(4), 854865. https://doi.org/10.1002/jaba.466

Kreipe, R. E., \& Palomaki, A. (2010). Beyond Picky Eating: Avoidant restrictive food intake disorder. Current Psychiatry Reports, 14(4), 421-431. https://doi.org/10.1007/s11920$\underline{012-0293-8}$

Krisch, K. A., Bandarian-Balooch, S., \& Neumann, D. L. (2018). Effects of extended extinction and multiple extinction contexts on ABA renewal. Learning and Motivation, 63(1), 1-10. https://doi.org/10.1016/j.lmot.2017.11.001

Liddon, C. J., Kelley, M. E., Rey, C. N., Liggett, A. P., \& Ribeiro, A. (2018). A translational analysis of $\mathrm{ABA}$ and $\mathrm{ABC}$ renewal of operant behavior. Journal of Applied Behavior Analysis, 51(4), 819-830. https://doi.org/10.1002/jaba.496

Mitteer, D. R., Greer, B. D., Fisher, W. W., Briggs, A. M., \& Wacker, D. P. (2018). A laboratory model for evaluating relapse of undesirable caregiver behavior. Journal of the Experimental Analysis of Behavior, 110(2), 252-266. https://doi.org/10.1002/jeab.462

Muething, C., Call, N., \& Pavlov, A. (2020). Prevalence of renewal of problem behavior during context changes. Journal of Applied Behavior Analysis. Advance online publication. https://doi.org/10.1002/jaba.672

Najdowski, A. C., Wallace, M. D., Penrod, B., Tarbox, J., Reagon, K., \& Higbee, T. S. (2008). Caregiver-conducted experimental functional analyses of inappropriate mealtime 
behavior. Journal of Applied Behavior Analysis, 41(3), 459-465.

https://doi.org/10.1901/jaba.2008.41-459

Nieto, J., Uengoer, M., \& Bernal-Gamboa. (2017). A reminder of extinction reduces relapse in an animal model of voluntary behavior. Learning \& Memory, 24(2), 76-80. https://doi.org/10.1101/1m.044495.116

Patel, M. R., Piazza, C. C., Santana, C. M., \& Volkert, V. M. (2002). An evaluation of food type and texture in the treatment of a feeding problem. Journal of Applied Behavior Analysis, 35(2), 183-186. https://doi.org/10.1901/jaba.2002.35-183

Peterson, K. M., Ibañez, V. F., Kirkwood, C. A., Crowley, J. G., \& Piazza, C. C. (2018). Assessment of Pediatric Feeding Disorders. In: Matson J. (eds) Handbook of Childhood Psychopathology and Developmental Disabilities Assessment. Autism and Child Psychopathology Series (pp. 415-431). Springer, Cham.

Piazza, C. C., Fisher, W. W., Brown, K. A., Shore, B. A., Patel, M. R., Katz, R. M., \& BlakelySmith, A. (2003). Functional analysis of inappropriate mealtime behaviors. Journal of Applied Behavior Analysis, 36(2), 187-204. https://doi.org/10.1901/jaba.2003.36-187

Piazza, C. C., Ibañez, V. F., Ney, H. M., Kirkwood, C. A., \& Crowley, J. G. (2017). Assessment and treatment of pediatric feeding disorders. Archives of Practitioner resources for applied behavior analysts. Kalamazoo, MI: Western Michigan University.

Podlesnik, C. A., \& DeLeon, I. G. (2015). Behavioral momentum theory: Understanding persistence and improving treatment. In F. D. DiGennaro Reed \& D. D. Reed (Eds.), Bridging the Gap Between Science and Practice in Autism Service Delivery (pp. 327351). New York: Springer.

Podlesnik, C. A., Kelley, M., E., \& Jimenez-Gomez, C., \& Bouton, M. E. (2017). Renewed behavior produced by context change and its implications for treatment maintenance: A review. Journal of Applied Behavior Analysis, 50(3), 675-697. https://doi.org/10.1002/jaba.400 
Pritchard, D., Hoerger, N., Mace, F. C., Penney, H., Harris, B., \& Eiri, L. (2016). Clinical translations of the ABA renewal model of treatment relapse. European Journal of Behavior Analysis, 17(2), 182-191. https://doi.org/10.1080/15021149.2016.1251144

Saini, V., \& Mitteer, D. R. (2019). A review of investigations of operant renewal with human participants: Implications for theory and practice. Journal of the Experimental Analysis of Behavior, 113(1), 105-123. https://doi.org/10.1002/jeab.562

Saini, V., Sullivan, W. E., Baxter, E. L., DeRosa, N. M., \& Roane, H. S. (2018). Renewal during functional communication training. Journal of Applied Behavior Analysis, 51(3), 603619. https://doi.org/10.1002/jaba.471

St. Peter Pipkin, C., Vollmer, T. R., \& Sloman, K. N. (2010). Effects of treatment integrity failures during differential reinforcement of alternative behavior: A translational model. Journal of Applied Behavior Analysis, 43(1), 47-70. https://doi.org/10.1901/jaba.2010.43$\underline{47}$

Sweeney, M. M., \& Shahan, T. A. (2013). Behavioral momentum and resurgence: Effects of time in extinction and repeated resurgence tests. Learning \& Behavior, 41(4), 414-424. https://doi.org/10.3758/s13420-013-0116-8

Tarquinio, D. C., Jones, M. C., Jones, K. L., \& Bird, L. M. (2012). Growth charts for 22q11 deletion syndrome. American Journal of Medical Genetics, 158A(11), 2672-2681. https://doi.org/10.1002/ajmg.a.35485

Todd, T. P., Winterbauer, N. E., \& Bouton, M. E. (2012). Effects of the amount of acquisition and contextual generalization on the renewal of instrumental behavior after extinction. Learning \& Behavior, 40(2), 145-157. https://doi.org/10.3758/s13420-011$\underline{0051-5}$

Todd, T. P. (2013). Mechanisms of renewal after the extinction of instrumental behavior. Journal of the Experimental Analysis of Behavior, 39(3), 193-207. https://doi.org/10.1037/a0032236 
Todd, T. P., Vurbic, D., \& Bouton, M. E. (2014). Mechanisms of renewal after the extinction of discriminated operant behavior. Journal of Experimental Psychology: Animal Learning and Cognition, 40(3), 355-368. https://doi.org//10.1037/xan0000021

Trask, S., Thrailkill, E., A., \& Bouton, M. E. (2017). Occasion setting, inhibition, and the contextual control of extinction in pavlovian and instrumental (operant) learning. Behavioural Processes, 137(7), 64-72. https://doi.org/10.1016/j.beproc.2016.10.003

University of Rochester Medical Center, Developmental and Behavioral Pediatric. Feeding Disorders: United States. https://www.urmc.rochester.edu/childrenshospital/developmental-disabilities/conditions/feeding-disorders.aspx. February 16, 2019.

Vaz, P. C. M., \& Piazza, C. C. (2010). Behavioral approaches to the management of pediatric feeding disorders. In C. Martin \& A. Southall (Eds.), Feeding problems in children: A practical guide for health professionals (pp. 53-73). Abingdon, U.K: Radcliffe.

Volkert, V. M., Patel, M. R., \& Peterson, K. M. (2016). Food refusal and selective eating. In J. K. Luiselli (Eds.), Behavioural Health Promotion and Intervention in Intellectual and Developmental Disabilities, Evidence-Based Practices in Behavioral Health (pp. 137161). Switzerland: Springer International Publishing.

Volkert, V. M., \& Piazza, C. C. (2012). Pediatric feeding disorders. In P. Sturmey \& M. Hersen (Eds.), Handbook of evidence-based practice in clinical psychology, Vol 1: Child and adolescent disorders (pp. 323-337). Hoboken, NJ, US: John Wiley \& Sons Inc. https://doi.org/10.1002/9781118156391.ebcp001013

Wacker, D. P., Harding, J. W., Berg, W. K., Lee, J. F., Schieltz, K. M., Padilla, Y. C., Nevin, J. A., \& Shahan, T. A. (2011). An evaluation of persistence of treatment effects during long-term treatment of destructive behavior. Journal of the Experimental Analysis of Behavior, 96(2), 261-282. https://doi.org/10.1901/jeab.2011.96-261 
Wathen, S. N. \& Podlesnik, C. A. (2018). Laboratory models of treatment relapse and mitigation techniques. Behavior Analysis: Research and Practice, 18(4), 362-387.

$\underline{\text { https://doi.org/10.1037/bar0000119 }}$ 